Published in final edited form as:

J Med Chem. 2016 October 13; 59(19): 8913-8923. doi:10.1021/acs.jmedchem.6b00801.

\title{
Structure-activity relationships and kinetic studies of peptidic antagonists of CBX chromodomains
}

\author{
Jacob I. Stuckey, Catherine Simpson, Jacqueline L. Norris-Drouin, Stephanie H. \\ Cholensky, Junghyun Lee, Ryan Pasca, Nancy Cheng, Bradley M. Dickson ${ }^{\dagger}$, Kenneth H. \\ Pearce, Stephen V. Frye*, and Lindsey I. James ${ }^{*}$ \\ Center for Integrative Chemical Biology and Drug Discovery, Division of Chemical Biology and \\ Medicinal Chemistry, UNC Eshelman School of Pharmacy, University of North Carolina at Chapel \\ Hill, Chapel Hill, North Carolina 27599, USA
}

\begin{abstract}
To better understand the contribution of methyl-lysine (Kme) binding proteins to various disease states, we recently developed and reported the discovery of $\mathbf{1}$ (UNC3866), a chemical probe that targets two families of Kme binding proteins, CBX and CDY chromodomains, with selectivity for CBX4 and -7. The discovery of $\mathbf{1}$ was enabled in part by the use of molecular dynamics simulations performed with CBX7 and its endogenous substrate. Herein, we describe the design, synthesis and structure-activity relationship studies that led to the development of $\mathbf{1}$ and provide support for our model of CBX7-ligand recognition by examining the binding kinetics of our antagonists with $\mathrm{CBX} 7$ as determined by surface-plasmon resonance.
\end{abstract}

\section{Graphical abstract}

\section{Introduction}

Controlled regulation of gene expression is essential for the proper development and function of multicellular organisms. Cell-type specific gene regulation is achieved by expression of specific transcription factors and through a complex language of chemical modifications to both DNA and histone proteins that are interpreted through the binding of

\footnotetext{
Corresponding Authors: ingerman@email.unc.edu (t: 919-962-4870) and svfrye@email.unc.edu (t: 919-843-5486).

${ }^{\dagger}$ Present Addresses: Center for Epigenetics, Van Andel Research Institute, Grand Rapids, Michigan 49503, USA

Author Contributions: Compound synthesis was performed by JIS, JL, and RP. AlphaScreen ${ }^{\circledR}$ assays were developed by CS, JIS and KHP, screening was performed by CS. JND and SHC expressed and purified all protein constructs. NC and JIS performed cell proliferation and compound permeability studies. JL performed pull-down experiments. JS and BDM designed all compounds. JIS performed all SPR studies. JS, KHP, SVF and LIJ planned and discussed all experiments. The manuscript was written through contributions of all authors. All authors have given approval to the final version of the manuscript.

Supporting Information. Descriptions of protein expression and purification, ITC experiments, cell culture and lysis conditions, general chemistry procedures, compound characterization data, and additional figures illustrating ITC results, pull-down results and SPR sensorgrams. This material is available free of charge via the Internet at http://pubs.acs.org
} 
various proteins and protein complexes. ${ }^{1}$ These 'reader' complexes then modulate the transcriptional outcome by controlling the accessibility of the underlying gene to transcription factors. ${ }^{2,3}$

One key chemical modification that regulates gene expression is the posttranslational methylation of histone lysine residues. ${ }^{2}$ The lysine $\varepsilon$-nitrogen can be mono-, di- or trimethylated (Kme1, Kme2 or Kme3, respectively). Methyl-lysine (Kme) reader proteins recognize Kme in a manner that is specific to the methylation state of the lysine and often to the sequence surrounding the modified lysine. ${ }^{3,4} \mathrm{Kme}$ readers bind methylated-lysine through an aromatic cage that engages the lysine side chain through cation- $\pi$ and van der Waals' interactions. The size and shape of the aromatic cage allows the Kme reader to discriminate between different methylation states, while the surrounding protein residues dictate sequence selectivity. ${ }^{3}$ Previous studies have characterized the ability of various Kme 1 and Kme2 reader proteins to accommodate non-natural methyl-lysine analogs in their aromatic cages: ${ }^{5-7}$ however, little is known about the preference of Kme3 reader proteins for different Kme mimetics. Initial efforts toward the discovery of Kme3 reader antagonists were focused on the development of peptidic inhibitors wherein the key Kme3 residue was maintained and potency was achieved through the variation of surrounding residues. ${ }^{8,} 9$

We recently reported the development of $\mathbf{1}$ (UNC3866), a peptide-based chemical probe that contains an unnatural diethyl-lysine in lieu of Kme 3 and selectively targets two families of Kme3 reader proteins (Figure 1). ${ }^{10}$ These two families of proteins belong to the chromodomain superfamily of Kme readers that are essential for proper genomic regulation in numerous organisms, spanning fungi, plants and animals. ${ }^{11}$ Compound $\mathbf{1}$ targets the Polycomb (Pc) CBX family of chromodomains ${ }^{4}$ and the lesser explored CDY family of chromodomains. ${ }^{12}$ In mammals, the Pc family of chromodomains consists of five proteins, CBX2, $-4,-6,-7$ and -8 . These proteins compete with each other for incorporation into Polycomb Repressive Complex 1 (PRC1) where they regulate numerous cellular processes including differentiation, growth and proliferation. ${ }^{13-17}$

Compound 1 is most potent for the chromodomains of CBX4 and $-7\left(K_{\mathrm{d}} \sim 100 \mathrm{nM}\right)$. It is 6 to 63 -fold selective for these chromodomains over the other CBX and CDY chromodomains. ${ }^{10}$ Interestingly, our selectivity studies revealed that $\mathbf{1}$ does not significantly interact $\left(K_{\mathrm{d}}>100 \mu \mathrm{M}\right)$ with the HP1 family of chromodomains (CBX1, -3 and -5$),{ }^{10}$ which share a high degree of structural similarity to the Pc family of chromodomains but are not associated with PRC1 ${ }^{4}$ Compound $\mathbf{1}$ was also highly selective versus $>250$ other molecular targets including chromatin regulatory proteins and a general pharmacology panel. Our previous studies characterized the effects of $\mathbf{1}$ in PC3 cells, an advanced stage metastatic prostate cancer model previously shown to have its growth regulated by CBX7. ${ }^{18}$ Consistent with CBX7 genetic manipulation, compound $\mathbf{1}$ is able to inhibit PC3 cell proliferation by inducing a senescent-like state. ${ }^{14,18}$ These studies suggest that Pc CBX antagonists may one day serve as a novel class of therapeutically useful agents.

Herein we report the structure-activity relationship (SAR) studies leading to the development of compound $\mathbf{1}$. We explored the effects of various modifications (Figure 2a) on the potency and selectivity versus three representative members of the Pc CBX 
chromodomains (CBX4, -7 and -8 ), with CBX4 and -7 being the most potent targets of $\mathbf{1}$, and CBX8 representing the remaining Pc CBX chromodomains (CBX2, -6, and -8), all of which display somewhat weaker affinity for $1 .{ }^{10}$ Compounds were also tested against one member of the CDY family of chromodomains, CDYL2, and a representative of the HP1 family of chromodomains, CBX5, in order to better understand the molecular determinants for selectivity between these three structurally related families of chromodomains. Additionally, kinetic studies with CBX7 provided key insights into the induced-fit recognition mechanism of these ligands by CBX7.

\section{Results and Discussion}

We first developed an AlphaScreen ${ }^{\circledR 19}$ assay for each of the Pc CBX and CDY chromodomains using His-tagged recombinant protein and 2 (UNC4195, Figure 2b), a biotinylated analog of $\mathbf{1}$, as the bait ligand. ${ }^{10}$ It should be noted that our previously reported AlphaScreen ${ }^{\circledR}$ studies with compound $\mathbf{1}$ were performed utilizing a similar assay with a biotinylated $\mathrm{H} 3 \mathrm{~K} 9 \mathrm{me} 3$ bait ligand and CBX7. The improved potency of $\mathbf{2}$ for our target chromodomains over the $\mathrm{H} 3 \mathrm{~K} 9 \mathrm{me} 3$ peptide allows us to reduce the amount of all reagents used in the assay and also reduce the tight binding limit. For CBX5, which does not appreciably bind to $\mathbf{2}$, we again utilized a biotinylated version of the endogenous substrate, $\mathrm{H} 3 \mathrm{~K} 9 \mathrm{me}^{4}$ (see Supplementary Table 1 for specific assay concentrations).

Compound $\mathbf{1}$ is based on a peptide fragment from the methyltransferase SETDB1 previously found to bind more tightly to Pc CBX chromodomains than H3K27me $3^{4}$. The initial SETDB1 sequence identified was a 10-residue peptide containing an A-L-Kme3-S motif. Therefore, our initial efforts sought to determine the minimal sequence from this peptide necessary for Pc CBX antagonism. Using solution-phase peptide synthesis and an Nterminal benzoyl cap to aid in purification (Supplementary Information, Synthetic Schemes 1 and 2), we generated a series of peptides based on the ALKme3S motif and found that, minimally, a 5 residue peptide (compound $\mathbf{5}$ ) is required for antagonism of the CBX chromodomains in our panel (Table 1, compounds 3-7). Compound 5 displayed modest affinity for CBX4 and -7, weak affinity for CBX8 and CBX5, and submicromolar potency for CDYL2. In contrast to the Pc CBX chromodomains, the chromodomain of CDYL2 maintains weak affinity for the shorter peptides, compounds 3, 4 and $\mathbf{7}$. Extending the peptide length to six residues resulted in compound $\mathbf{6}$ which displays somewhat reduced affinity for each panel member relative to $\mathbf{5}$, suggesting that there are subtle differences in the way these different chromodomains recognize the $\mathrm{N}$-terminus of these molecules. Peptide 7 binding affinities were consistent with previous observations that binding of CBX chromodomains requires the presence of a serine $\mathrm{C}$-terminal to the methyl-lysine. ${ }^{4,8}$

Concomitantly with our studies to investigate the minimal length requirements for chromodomain engagement, we had observed robust binding of compound 6 with CBX7 in isothermal titration calorimetry (ITC) studies $\left(K_{\mathrm{d}}=10.3 \pm 0.9 \mu \mathrm{M}\right.$, Supplementary Figure 1). Therefore, we sought to use $\mathbf{6}$ as a scaffold for further SAR exploration in parallel. $N_{\mathrm{a}^{-}}$ methylation has previously been shown to improve the membrane permeability of peptides ${ }^{20}$ so we explored the effect of methylating select backbone amides of $\mathbf{6}$ on chromodomain binding (peptides 8-10). Methylation of the alanine amide nitrogen (8) resulted in loss of 
binding to all chromodomains tested. Methylation of the phenylalanine amide nitrogen (9) selectively bound to CDYL2 while methylation of the glycine amide in compound $\mathbf{1 0}$ had little effect on binding to any of our panel members when compared to the corresponding unmethylated analog (6). These results combined with prior structural studies ${ }^{4,21}$ suggest that the alanine $\mathrm{N}-\mathrm{H}$ of these peptides makes a critical hydrogen bond that is necessary for binding to chromodomains. The amide N-H of the Phe residue of these and related peptides does not appear to be positioned to form any hydrogen bonds when bound to Pc chromodomains, ${ }^{10,21}$ suggesting that this modification may instead introduce a steric clash. Finally, when combined with prior structural studies, ${ }^{4,10,21}$ these results indicate that the benzamide cap of both $\mathbf{6}$ and $\mathbf{1 0}$ is solvent-exposed when bound to CBX4, -7 or CDYL2 and the Gly amide is likely not making any significant protein contacts.

In parallel with our studies in Table 1, we focused on finding a suitable replacement for the Kme3 of $\mathbf{6}$ in an effort to increase passive membrane permeability and enhance stability toward demethylase enzymes ${ }^{22}$ (Table 2). Consistent with previous studies, the nonmethylated lysine analog of $\mathbf{6}$, compound 11, displayed no antagonism of any of the chromodomains in the panel. ${ }^{4}$ We performed a series of reductive aminations on $\mathbf{1 1}$ (Supplementary Information, Synthetic Scheme 3) to explore the effect of replacing the quaternary amine with bulky secondary amines (compounds 12-16) and in general, found that the CBX chromodomains have limited affinity for these secondary amines, while the CDYL2 chromodomain is able to bind these ligands with modest affinity. For example, simple isopropylation of the lysine (12) showed no affinity for CBX chromodomains but single-digit micromolar affinity for CDYL2. Incorporation of a cyclopentyl or cyclohexyl (compounds 13 and 14) did not recover CBX activity. Interestingly, fusion of the 5- and 6membered rings in norbornyl-lysine (15) showed weak antagonism of CBX7 and equipotent antagonism of CDYL2 when compared to cyclopentyl alone (13). Adamantyl substitution (16) resulted in no measurable CBX7 activity and CDYL2 activity comparable to the cyclohexyl compound (14), indicating that increasing the size of the fused ring system beyond norbornyl (15) is not beneficial to chromodomain binding. Taken together, this data suggests that CDYL2 is more accommodating to various secondary amine Kme3 replacements than the $\mathrm{CBX}$ chromodomains.

We next focused on the effects of tertiary substitutions at the $\varepsilon$-nitrogen of the lysine residue. Unsurprisingly, ${ }^{4,12}$ we found that dimethylation (17) reduced the activity for both CBX7 and CDYL2 about 3-fold relative to the analogous Kme3 compound (6). However, incorporation of a slightly larger diethyl-lysine as in compound $\mathbf{1 8}$ resulted in similar affinities for all chromodomains as compound $\mathbf{6}$, albeit with slighted improved affinity for CBX8. Encouragingly, this supported the notion that a quaternary amine was not likely to be required for potent $\mathrm{CBX}$ chromodomain binding. To generate cyclic tertiary amines as in compounds 19-21, we reacted 11 with a series of dibromoalkanes (Supplementary Information, Synthetic Scheme 3). Cyclization of the diethyl-lysine to generate the pyrrolidinyl derivative, 19, resulted in a reduction in potency across the panel, while increasing the ring size to a piperidinyl group (20) or a 7-membered azepane (21) also did not appear to be beneficial. Incorporation of a bulkier dicyclobutyl-lysine (22) also did not result in any significant enhancements in potency. 
We were intrigued by the ability of the norbornyl functionality to weakly interact with CBX7 and more potently with CDYL2. It is possible that binding of this functionality to the aromatic cage could sterically hinder any cation- $\pi$ interactions between the cage residues and the $\varepsilon$-nitrogen, relying less on the presence of a basic nitrogen for binding. We had simultaneously prepared an acetylated version of $\mathbf{1 5}$ (compound 23) and although potency was reduced approximately 7-fold for CDYL2, the presence of a basic nitrogen does not appear to be an absolute requirement for CDYL2 binding. We further interrogated the nature of the cation- $\pi$ interaction at this position by synthesizing the more strongly basic tetramethylguanidine derivative by reacting $\mathbf{1 1}$ with TBTU in the absence of a carboxylic acid to give compound 24, and additionally prepared dibasic compounds 25 and 26 via reductive amination (Supplementary Information, Synthetic Scheme 3). All three of these compounds showed no activity for the CBX chromodomains and weakened affinity for CDYL2. Taken together, the SAR data resulting from modification of the lysine indicates that the presence of a quaternary amine is not required for engagement of our chromodomain panel; however, only the diethyl-lysine containing compound (18) demonstrates a binding profile comparable to that of $\mathbf{6}$. Substantially improving potency for Pc CBX chromodomains through modification of the lysine amine presents a significant challenge, but may be a more viable strategy for CDY chromodomain ligand optimization.

Our previously described in silico studies ${ }^{10}$ on the interaction of CBX7 and an H3K9me3 peptide provided insight into the mechanism of induced-fit recognition of Kme3 peptides by CBX7. These studies suggested that the chromodomain of CBX7 first recognizes the Nterminal cap residue at the (-4) position from the methyl-lysine, allowing the peptide to engage the chromodomain and leading $\mathrm{CBX} 7$ to close around the histone and engage the Kme3 with its newly formed aromatic cage. ${ }^{10}$ This induced-fit binding mechanism supports peptidomimetics as a likely choice for CBX7 inhibitors and the absence of a preformed aromatic cage makes the discovery of traditional small molecule inhibitors a significant challenge. Previous studies from the Zhou lab have reported weak, non-peptidic small molecule CBX7 ligands; however, SAR studies around these molecules struggled to produce significant improvements in potency. ${ }^{21,23}$

Our in silico studies led us to hypothesize that the potency of our peptidic antagonists could be improved through modification of the N-terminus. Diethyl-lysine was chosen from our studies in Table 2 as an optimal Kme3 replacement and hence was incorporated in future antagonists while we varied the N-terminus (Table 3, compounds 1 and 27-41; Supplementary Information, Synthetic Schemes 1 and 4). Our studies in Table 1 indicated that the glycine residue of $\mathbf{6}$ was dispensable, which led us to prepare compound $\mathbf{2 7}$, which is the diethyl-lysine analog of compound $\mathbf{5}$. This compound overall showed similar or improved activities toward each of the chromodomains bound by $\mathbf{5}$, further confirming that diethyl-lysine is a suitable Kme3 replacement in the context of this shorter peptide. Interestingly, 27 demonstrates measurable affinity for CBX5 in contrast to compound $\mathbf{1 8}$, providing initial evidence that the aromatic cage of CBX5 can also accommodate nonnatural lysine analogs.

We next began exploring substituents on the N-terminal aromatic ring of $\mathbf{2 7}$ by placing a methyl substituent at the ortho-, meta- and para-positions. Methylation at the ortho-position 
(28) of the ring dramatically reduced potency for all chromodomains except for CDYL2, causing a 20-fold decrease in binding for CBX7 relative to compound 27. Affinity for all chromodomains except CBX5 is recovered to levels comparable to $\mathbf{2 7}$ when the methyl group is shifted to the meta-position (29). Installation of para-Me (30) results in further improvements in binding to CBX7 and CBX4, with submicromolar affinities in each case. Interestingly, CBX8 did not display the same preference as CBX4 and -7 for $\mathbf{3 0}$ over $\mathbf{2 9}$, while methylation had little to no effect on CDYL2 binding.

Having determined that, in general, alkylation is preferred at the para-position by Pc chromodomains, we next explored the effect of increasing steric bulk at this position (compounds 31-32 and 1). Overall, increasing lipophilicity had opposing effects on the affinity for Polycomb vs HP1 chromodomains. Introduction of a para-ethyl or para-isopropyl substituent as in compounds $\mathbf{3 1}$ and $\mathbf{3 2}$, respectively, increased the affinity $\sim 2-5$ fold for CBX4, -7 , and -8 , as well as CDYL2, relative to $\mathbf{3 0}$, while the affinity for CBX5 was largely unchanged or slightly weakened. $t$-Butyl substitution (1) further improved potency for CBX4 and -7 while having no significant effect on the affinity for CBX8 and causing a loss of detectable CBX5 affinity. Taken together, these data indicate that potency and selectivity can be tuned by systematically adjusting both the position of alkylation and degree of lipophilicity on the benzoyl cap at the $\mathrm{N}$-terminus. These studies enabled us to dramatically improve the potency of our compounds for $\mathrm{CBX} 4$ and - 7, leading to compounds with $\mathrm{IC}_{50}$ values of less than $100 \mathrm{nM}$. These results also provided strong support for our molecular dynamics studies, which suggested that initial recognition of histones occurs through hydrophobic contacts formed between $\mathrm{CBX} 7$ and the histone side chain at this position. ${ }^{10}$

We also evaluated the effect of incorporating electron-withdrawing groups at the paraposition of the $\mathrm{N}$-terminal phenyl cap with compounds $\mathbf{3 3}$ and $\mathbf{3 4}$. A methyl ester at the para-position (33) resulted in an approximately 15 -fold loss in potency for both CBX4 and -7 relative to compound $\mathbf{1}$, but only an approximately 3 -fold loss in potency for CBX8, further suggesting that CBX4 and -7 depend more strongly on the presence of bulky alkyl substituents at this position. The strongly electron-withdrawing trifluoromethyl substituent of $\mathbf{3 4}$ also weakened binding to the Pc chromodomains, although to a lesser extent than compound 33. CDYL2 affinity is largely unaffected by replacement of para-alkyl substituents with electron-withdrawing groups, consistent with the generally less stringent binding preferences of CDYL2. Inclusion of an electron-donating group (35) also did not further improve binding to Pc CBX chromodomains, indicating that alkyl substituents at this position are preferred. In contrast, these results suggest that $\mathrm{CBX} 5$ may prefer less sterically demanding functional groups at the $\mathrm{N}$-terminus.

We next investigated the importance of the spacing between the $\mathrm{N}$-terminal hydrophobic group and the rest of the peptide. As can be seen with compound 36, addition of a single methylene group relative to compound $\mathbf{1}$ resulted in an approximately 100-fold loss of potency for CBX4 and -7 and almost a 20-fold loss for CBX8, while CDYL2 was largely unable to distinguish between the $t$-butyl phenyl and $t$-butyl benzyl moieties. Additionally, larger bicyclic aromatic capping groups with a methylene spacer (37 and 38) were also less well tolerated by $\mathrm{CBX} 4,-7$ and -8 . Consistent with prior results, the increased steric demand of these functionalities was detrimental to CBX5 activity. 
Our studies in Table 2 indicated that the tolerance of Pc CBX chromodomains to unnatural Kme analogs is fairly limited, yet we were interested in exploring a small number of lysine modifications, as well as the native Kme 3 residue, in the context of our more potent peptide scaffold. We generated the Kme 3 analog of $\mathbf{1}$, compound $\mathbf{3 9}$, and two asymmetric tertiary amine derivatives, compounds $\mathbf{4 0}$ and $\mathbf{4 1}$. The isopropyl group of $\mathbf{4 0}$ and $\mathbf{4 1}$ was hypothesized to make additional contacts with the aromatic cage of the Pc CBX proteins and to more completely fill the cage based on the crystal structures of $\mathbf{1}$ bound to these chromodomains (Figure 3a). Mirroring prior results, the Kme 3 containing compound (39) was about equipotent to 1, while the bulkier lysine mimetics of compounds $\mathbf{4 0}$ and $\mathbf{4 1}$ also did not appear to pick up any additional interactions to further improve potency.

Previous studies from the Hof group found that the residue at the (-2) position from the modified lysine can have profound effects on the selectivity and potency of peptide antagonists for Pc CBX chromodomains. ${ }^{9}$ We explored this in the context of our antagonists by testing the effect of increasing the size of the alkyl side chain at this position (Table 4, compounds 42 and 43; Supplementary Information, Synthetic Scheme 4). We found that replacing the methyl side chain of alanine with an ethyl or isopropyl group reduced affinity for CBX4, -7 , and CDYL2 while slightly increasing the affinity for CBX8. This was not surprising as previously solved co-crystal structures with 1 revealed that the pocket occupied by this alanine side chain is larger in CBX8 than in CBX4 and -7 owing to substitution of Val13 in CBX4/7 with Ala13 in CBX8 (Figure 3b). ${ }^{10}$ The increased potency and modest selectivity of $\mathbf{4 3}$ for CBX8 may make it a useful starting point for the development of more potent and selective CBX8 compounds.

Modifications to the C-terminus of compound 1 (compounds 44-46; Supplementary Information, Synthetic Schemes 4 and 5), were reasonably well tolerated. While the methyl ester of $\mathbf{1}$ is the preferred functionality at this position, replacement with the corresponding acid (44) or dimethyl amide (45), or removal of the C-terminal carbonyl entirely (46), resulted in a mere 2- to 4-fold reduction in potency for Pc CBX chromodomains. Interestingly, compound $\mathbf{4 4}$ resulted in a slightly larger reduction in potency for CDYL2 (5fold), suggesting that additional exploration of the C-terminus of these molecules may be useful for further tuning selectivity.

The observed tolerance to modifications at the $\mathrm{C}$-terminus previously led us to generate a biotinylated analog of $\mathbf{1}$ (compound 2). This tool compound showed minimal loss in potency for CBX7, and importantly was used to evaluate the selectivity of compound $\mathbf{1}$ more broadly and served as a key chemiprecipitation reagent to demonstrate cellular target engagement. ${ }^{10}$ The co-crystal structures of the Pc CBX proteins with compound 1 revealed that the phenylalanine of $\mathbf{1}$ is largely solvent exposed (Figure 3c); therefore, we hypothesized that this was an additional position we could use for tagging to access additional tool compounds. An amine functional group was installed on the phenylalanine side chain, and as expected from the crystallographic information, compound $\mathbf{4 7}$ displayed similar affinities to $\mathbf{1}$ for each of the chromodomains in our panel. Further, because the boc group of $\mathbf{4 8}$ does not interfere with binding, we reasoned that this would be a suitable location to place larger chemical moieties. To this end, we generated a biotinylated derivative of $\mathbf{4 7}$ (biotin-47; Supplementary Information, Synthetic Scheme 6) via modification of the free amine. 
Compound 2 was previously shown to selectively chemiprecipitate CBX4, -7 and -8 , but not CBX2 or -6 from PC 3 cell lysates, despite having demonstrated affinity for these proteins. ${ }^{10}$ Similarly, we found that biotin-47 was unable to pulldown CBX2 and -6, while it also lost the ability to pull-down CBX7, indicating that pull-down of these endogenous proteins from cell lysates is highly sensitive to the positioning of the chemical tag (Supplementary Figure 2). It is possible that biotinylation of the ligand produces a steric clash in the context of specific intact PRC1 complexes that can't be anticipated from CBX-only co-crystal structures.

While modifications to the lysine side chain did not improve the in vitro potency of our compounds, we sought to compare the cellular activity of compound 39 (containing Kme3) to compound $\mathbf{1}$ since we initially hypothesized that replacement of the Kme3 would improve passive membrane permeability. We first measured the intracellular concentration of $\mathbf{3 9}$. Treatment at a $30 \mu \mathrm{M}$ extracellular dose of $\mathbf{1}$ for 24 hours results in an intracellular concentration of $1.4 \pm 0.3 \mu \mathrm{M}$ in PC 3 cells. ${ }^{10}$ Under the same conditions, we observed an intracellular concentration of $0.61 \pm 0.2 \mu \mathrm{M}$ of compound 39. It was surprising that incorporation of trimethyl-lysine resulted in only an approximately 2-fold decrease in the permeability of the compound, and we were intrigued to investigate whether $\mathbf{3 9}$ was functionally available for chromodomain binding in cells since it is known that highly charged compounds may accumulate in endosomes and lysosomes. ${ }^{24,25}$

Previously, we have shown that 1 inhibits PC 3 cell proliferation through antagonism of polycomb CBX chromodomains. In order to assess the effect of Kme3 substitution on functional activity, we compared the anti-proliferative effects of $\mathbf{1}$ and $\mathbf{3 9}$ to our negative control analog of 1, compound 49 (UNC4219). Compound 49 contains a single backbone methylation which ablates its ability to interact with chromodomains. ${ }^{10}$ Despite having nearly identical in vitro potencies for CBX chromodomains as $\mathbf{1}$ and only a 2-fold reduction in permeability, compound $\mathbf{3 9}$ had no effect on PC3 cell proliferation, analogous to the negative control (Figure 4), supporting our hypothesis that Kme3 replacement is required for cellular activity. We suspect that the lack of biological activity observed upon treatment with compound 39 may be a result of endosomal and lysosomal trapping of the molecule. ${ }^{24,25}$

As discussed previously, our proposed binding model involves an induced-fit mode of recognition in which the $\mathrm{N}$-terminus of these ligands makes the first specific contact with CBX7 and that initial hydrophobic interaction nucleates a series of backbone hydrogen bonds, allowing CBX7 to close and the aromatic cage to form to generate the stably bound state. While we were able to apply this model to develop more potent chromodomain antagonists, largely through modification of the $\mathrm{N}$-terminal aromatic capping group, we were motivated to further interrogate the validity of the model. We therefore turned to surface-plasmon resonance (SPR) studies with CBX7 and a subset of our compounds that differ at the $\mathrm{N}$-terminus in order to determine if the binding kinetics could provide any evidence to further support this induced-fit hypothesis.

When first comparing the SPR data for compound $\mathbf{1 8}$ (Figure 5 and Supplementary Figure 3), which contains the larger benzoyl glycine $\mathrm{N}$-terminus, to the ortho-Me phenyl derivative (28), both of which have similar $\mathrm{IC}_{50}$ 's for $\mathrm{CBX} 7$, we observed that the $k_{\text {on }}$ for compound 28 
was slightly faster but that the $k_{\text {off }}$ increased in a proportional manner, resulting in comparable affinities. When the methyl group of compound $\mathbf{2 8}$ is shifted to the metaposition (compound 29), the higher potency observed for this compound is largely driven by a significant increase in $k_{o n}$. Moving the methyl substituent to the para-position (compound 30) did not substantially change the $k_{\text {on }}$ relative to compound 29 but did slightly improve $k_{\text {off }}$, resulting in the 3 -fold improvement in CBX7 potency for $\mathbf{3 0}$ over $\mathbf{2 9}$ observed in AlphaScreen ${ }^{\circledR}$. Inclusion of a para-t-butyl moiety in $\mathbf{1}$ further improves both $k_{\text {on }}$ and $k_{\text {off }}$, resulting in one of our most potent CBX7 inhibitors to date.

We were intrigued by the fact that the majority of the potency improvements resulting from modification of the $\mathrm{N}$-terminus were driven primarily by enhanced association rates instead of decreased dissociation rates. ${ }^{26}$ Based on molecular dynamics simulations, we believe that $\mathrm{N}$-terminal portion of these molecules forms the first stable interaction with CBX7, which is consistent with modifications at this position primarily affecting the association kinetics.

More specifically, the improvement in association kinetics may result from a reduction in the conformational flexibility at the $\mathrm{N}$-terminus, thereby increasing the likelihood of a productive binding collision between CBX7 and the antagonist. SPR results with CBX7 and compound 36, which is approximately 100 -fold less potent than $\mathbf{1}$ for CBX7, demonstrate that insertion of a flexible methylene linker primarily reduced $k_{\text {on }}$ while $k_{\text {off }}$ was minimally affected. Finally, analysis of the $k_{\text {on }}$ and $k_{\text {off }}$ rates of these select compounds with respect to their $K_{\mathrm{d}}$ 's (Figure $5 \mathrm{c}$ ) revealed that the binding is better correlated with $k_{\text {On }}$ as opposed to $k_{\text {off }}$, providing even stronger support for this hypothesis.

\section{Conclusions}

The studies presented herein build on previous work investigating structure-activity relationships of peptide-based antagonists for Pc CBX chromodomains. ${ }^{8-10}$ In particular, we performed the first systematic effort to replace Kme3 with non-natural lysine derivatives and found that tertiary amine Kme3 mimetics are not only tolerated, but are able to maintain similar affinities for chromodomains. Replacement of Kme3 was also found to be critical for cellular activity of these ligands.

A thorough examination of the $\mathrm{N}$-terminus of our inhibitors has also begun to establish the requirements for optimizing potency and selectivity for the Pc, HP1 and CDY families of chromodomains. While CDYL2 appears to be much more tolerant of various modifications at this position in our studies, CBX chromodomains show a strong preference for alkyl substituents located at the para-position of an N-terminal benzoyl cap. Pc chromodomains tend to prefer larger alkyl substituents at this position while the CBX5 chromodomain interacts more favorably with smaller substituents or an unmodified benzoyl group at this position. Further studies will help to elaborate on the requirements for CBX selectivity at this position, and we are currently pursuing alternative approaches toward the discovery and optimization of selective chromodomain ligands via an affinity-based, one-bead onecompound, target class screening strategy. ${ }^{27}$

Our SPR studies have provided strong support for the hypothesized induced-fit binding mechanism for these antagonists with CBX7. These studies have revealed what is, to our 
knowledge, the first example of a protein-ligand system with an exploitable SAR to control potency via optimization of association kinetics. Further, these studies have demonstrated that the residence time of compound $\mathbf{1}$ on CBX7 is quite short ( $\sim 100$ seconds). There are an increasing number of examples where the cellular efficacy of drugs and probes are better correlated with the residence time than the binding constant. ${ }^{26}$ Therefore, we are currently working to apply our knowledge of the binding pathway to increase the residence time of these ligands in order to assess whether this will improve their cellular and in vivo efficacy.

\section{Experimental Section}

\section{Expression constructs}

The chromodomains of CBX4 (residues 8-65 of NP_003646), CBX5 (residues 18-75 of NP_036429) and CBX8 (residues 8-61 of NP_065700) were expressed with N-terminal Histags in pET28 expression vectors. The chromodomain of CBX7 (residues 8-62 of NP_783640) and CDYL2 (residues 1-75 of NP_689555) were expressed with a C-terminal His-tag in a pET30 expression vector.

\section{AlphaScreen ${ }^{\circledR}$ procedure and conditions}

The AlphaScreen ${ }^{\circledR}$ assay was generally performed as previously described. ${ }^{19}$ In brief, compound plates $(1 \mu \mathrm{L}$ at $10 \mathrm{mM}$ highest concentration; 3-fold, 10-point dilutions in DMSO) were diluted in $1 \times$ assay buffer ( $20 \mathrm{mM}$ TRIS pH 7.5, $75 \mathrm{mM} \mathrm{NaCl}, 2 \mathrm{mM}$ DTT and $0.05 \%$ Tween-20) to $1 \mathrm{mM}$ using a Multimek robotic pipettor (Nanoscreen) and $1 \mu \mathrm{L}$ was spotted into the wells of 384-well low-volume Proxiplates (PerkinElmer). To these plates $9 \mu \mathrm{L}$ of protein-peptide mix in $1 \times$ assay buffer was added by Multidrop (Thermo) to bring the final compound concentration to $100 \mu \mathrm{M}$ and incubated for $30 \mathrm{~min}$ at room temperature. Next, $2 \mu \mathrm{L}$ of a 1:1 mixture of streptavidin-conjugate donor and nickel-chelate or a-GST acceptor beads ( $45 \mu \mathrm{g} / \mathrm{mL}$ in $1 \times$ assay buffer) were added and the plates were allowed to incubate for an additional $30 \mathrm{~min}$ in the dark at room temperature. After incubation, the plates were read on an EnVision multi-label reader equipped with an HTS AlphaScreen laser (Perkin Elmer). Dose-response curves were fit using a 4-parameter or 3parameter fixed top binding equation.

\section{Compound permeability studies}

PC3 cells were cultured until reaching $\sim 80 \%$ confluency in a T75 cell culture flask. The media was exchanged with $10 \mathrm{~mL}$ fresh media (0.3\% DMSO) containing $30 \mu \mathrm{M}$ compound. Cells were incubated with compound for 24 hours. After 24 hours, cells were washed with PBS, trypsinized, and wash again with PBS. The resulting cell pellets (3) were flash frozen with liquid nitrogen and submitted to Drumetix Laboratories (Greensboro, NC) for analysis by LC-MS/MS.

\section{Pull-down studies}

Cells were cultured on a T175 tissue culture flask until reaching $\sim 80-90 \%$ confluency. Following trypsinization, the pellet was washed twice with PBS and lysed with $500 \mu \mathrm{L}$ of lysis buffer. When applicable, vehicle control or $100 \mu \mathrm{M}$ of compound $\mathbf{1}$ was added to an aliquot of $1000 \mu \mathrm{g}$ of total protein and diluted to $500 \mu \mathrm{L}$ with $20 \mathrm{mM}$ Tris $(\mathrm{pH} 8) / 150 \mathrm{mM}$ 
$\mathrm{NaCl} / 0.1 \%$ Tween-20 (TBST). Pull-down reagents were bound to Streptavidin M-280 Dynabeads ${ }^{\circledR}$ by rotating $30 \mu \mathrm{L}$ of beads with a 20 -fold excess of pull-down reagent for 30 minutes at room temperature in. Unbound pull-down reagent was then removed and the beads were washed $3 \times$ with $200 \mu \mathrm{L}$ of TBST. The lysate was then transferred to an Eppendorf tube containing $30 \mu \mathrm{L}$ of Streptavidin M-280 Dynabeads ${ }^{\circledR}$ that had been prebound to biotin-46. The mixture was rotated for $2 \mathrm{hr}$ at $4^{\circ} \mathrm{C}$. The depleted lysate was removed and the beads were washed $3 \times$ with $300-500 \mu \mathrm{L}$ of TBST. The beads were resuspended with $30 \mu \mathrm{L}$ of $1 \times$ Laemmli sample buffer and heated at $95^{\circ} \mathrm{C}$ for 3 minutes. Fifteen microliters of the sample was then loaded into a gel and analyzed via western blotting using the appropriate primary antibody and detected using a LI-COR Odyssey® instrument and the appropriate fluorescent secondary antibodies (diluted 1:10, 000 in PBST). One percent of input was used for western blotting of all proteins.

\title{
Cell Proliferation Experiments
}

PC3 cells were seeded at 400 cells/well into 96-well plates. Cells were allowed to adhere overnight. The media (DMEM supplemented with $10 \%$ FBS) was then exchanged with fresh media containing DMSO, or $30 \mu \mathrm{M}$ of the indicated compound. On day three, the cells were fixed with ice-cold methanol for $30 \mathrm{sec}$. and rehydrated with PBS. Nuclei of the cells were stained with DAPI $(0.05 \mu \mathrm{g} / \mathrm{ml})$ and numerated using High Content Microscopy (Array Scan ${ }^{\mathrm{TM}}$ High Content Analysis, Thermo Fisher \#NX10002L).

\section{Surface Plasmon Resonance Experiments}

SPR experiments were performed on a ProteOn ${ }^{\mathrm{TM}}$ XPR36 Interaction Array System. Cterminally His-tagged CBX7 was immobilized using a ProteOn ${ }^{\mathrm{TM}}$ HTE sensor chip. Compounds were flowed at a rate of 50-100 $\mu \mathrm{L} /$ minute with a contact time of 150 seconds. Dissociation steps were run for 300 seconds at a flow rate of $100 \mu \mathrm{L} /$ minute. Double referencing subtraction was done with buffer and protein blank channels to account for nonspecific binding to the sensor chip. Sample Sensorgrams are shown in Supplementary Figure 3.

\section{Supplementary Material}

Refer to Web version on PubMed Central for supplementary material.

\section{Acknowledgments}

\begin{abstract}
We would like to acknowledge Ashutosh Tripathy (UNC Chapel Hill) for his assistance with the SPR experiments in this work. We thank Noah Sciaky (UNC Chapel Hill) for his help with the high content microscopy experiments. We would also like to acknowledge Mark Bedford (MD Anderson) for the CDYL2 chromodomain constructs and Cheryl Arrowsmith (SGC Toronto) for the CBX chromodomain constructs.

Funding Sources: The research described here was supported by the National Institute of General Medical Sciences, US National Institutes of Health (NIH, grant R01GM100919), the Carolina Partnership and the University Cancer Research Fund, University of North Carolina at Chapel Hill.
\end{abstract}

\section{References}

1. Strahl BD, Allis CD. The language of covalent histone modifications. Nature. 2000; 403:41-45. [PubMed: 10638745] 
2. Arrowsmith CH, Bountra C, Fish PV, Lee K, Schapira M. Epigenetic protein families: a new frontier for drug discovery. Nat Rev Drug Discovery. 2012; 11:384-400. [PubMed: 22498752]

3. Yun M, Wu J, Workman JL, Li B. Readers of histone modifications. Cell Res. 2011; 21:564-578. [PubMed: 21423274]

4. Kaustov L, Ouyang H, Amaya M, Lemak A, Nady N, Duan S, Wasney GA, Li Z, Vedadi M, Schapira M, Min J, Arrowsmith CH. Recognition and specificity determinants of the human cbx chromodomains. J Biol Chem. 2011; 286:521-529. [PubMed: 21047797]

5. James LI, Korboukh VK, Krichevsky L, Baughman BM, Herold JM, Norris JL, Jin J, Kireev DB, Janzen WP, Arrowsmith CH, Frye SV. Small-molecule ligands of methyl-lysine binding proteins: optimization of selectivity for L3MBTL3. J Med Chem. 2013; 56:7358-7371. [PubMed: 24040942]

6. Herold JM, James LI, Korboukh VK, Gao C, Coil KE, Bua DJ, Norris JL, Kireev DB, Brown PJ, Jin J, Janzen WP, Gozani O, Frye SV. Structure-activity relationships of methyl-lysine reader antagonists. Med Chem Commun. 2012; 3:45-51.

7. Perfetti MT, Baughman BM, Dickson BM, Mu Y, Cui G, Mader P, Dong A, Norris JL, Rothbart SB, Strahl BD, Brown PJ, Janzen WP, Arrowsmith CH, Mer G, McBride KM, James LI, Frye SV. Identification of a fragment-like small molecule ligand for the methyl-lysine binding protein, 53BP1. ACS Chem Biol. 2015; 10:1072-1081. [PubMed: 25590533]

8. Simhadri C, Daze KD, Douglas SF, Quon TT, Dev A, Gignac MC, Peng F, Heller M, Boulanger MJ, Wulff JE, Hof F. Chromodomain antagonists that target the polycomb-group methyllysine reader protein chromobox homolog 7 (CBX7). J Med Chem. 2014; 57:2874-2883. [PubMed: 24625057]

9. Milosevich N, Gignac MC, McFarlane J, Simhadri C, Horvath S, Daze KD, Croft CS, Dheri A, Quon TT, Douglas SF, Wulff JE, Paci I, Hof F. Selective inhibition of CBX6: a methyllysine reader protein in the polycomb family. ACS Med Chem Lett. 2016; 7:139-144. [PubMed: 26985288]

10. Stuckey JI, Dickson BM, Cheng N, Liu Y, Norris JL, Cholensky SH, Tempel W, Qin S, Huber KG, Sagum C, Black K, Li F, Huang XP, Roth BL, Baughman BM, Senisterra G, Pattenden SG, Vedadi M, Brown PJ, Bedford MT, Min J, Arrowsmith CH, James LI, Frye SV. A cellular chemical probe targeting the chromodomains of Polycomb repressive complex 1. Nat Chem Biol. 2016; 12:180 187. [PubMed: 26807715]

11. Eissenberg JC. Structural biology of the chromodomain: form and function. Gene. 2012; 496:6978. [PubMed: 22285924]

12. Fischle W, Franz H, Jacobs SA, Allis CD, Khorasanizadeh S. Specificity of the chromodomain Y chromosome family of chromodomains for lysine-methylated ARK(S/T) motifs. J Biol Chem. 2008; 283:19626-19635. [PubMed: 18450745]

13. Vandamme J, Volkel P, Rosnoblet C, Le Faou P, Angrand PO. Interaction proteomics analysis of polycomb proteins defines distinct PRC1 complexes in mammalian cells. Mol Cell Proteomics. 2011; 10 M110.002642-1-23.

14. Gil J, Bernard D, Martinez D, Beach D. Polycomb CBX7 has a unifying role in cellular lifespan. Nat Cell Biol. 2004; 6:67-72. [PubMed: 14647293]

15. Ren X, Vincenz C, Kerppola TK. Changes in the distributions and dynamics of polycomb repressive complexes during embryonic stem cell differentiation. Mol Cell Biol. 2008; 28:28842895. [PubMed: 18316406]

16. O'Loghlen A, Munoz-Cabello AM, Gaspar-Maia A, Wu HA, Banito A, Kunowska N, Racek T, Pemberton HN, Beolchi P, Lavial F, Masui O, Vermeulen M, Carroll T, Graumann J, Heard E, Dillon N, Azuara V, Snijders AP, Peters G, Bernstein E, Gil J. MicroRNA regulation of Cbx7 mediates a switch of Polycomb orthologs during ESC differentiation. Cell Stem Cell. 2012; 10:3346. [PubMed: 22226354]

17. Klauke K, Radulovic V, Broekhuis M, Weersing E, Zwart E, Olthof S, Ritsema M, Bruggeman S, Wu X, Helin K, Bystrykh L, de Haan G. Polycomb Cbx family members mediate the balance between haematopoietic stem cell self-renewal and differentiation. Nat Cell Biol. 2013; 15:353362. [PubMed: 23502315]

18. Bernard D, Martinez-Leal JF, Rizzo S, Martinez D, Hudson D, Visakorpi T, Peters G, Carnero A, Beach D, Gil J. CBX7 controls the growth of normal and tumor-derived prostate cells by repressing the Ink4a/Arf locus. Oncogene. 2005; 24:5543-5551. [PubMed: 15897876] 
19. Wigle TJ, Herold JM, Senisterra GA, Vedadi M, Kireev DB, Arrowsmith CH, Frye SV, Janzen WP. Screening for inhibitors of low-affinity epigenetic peptide-protein interactions: an AlphaScreenbased assay for antagonists of methyl-lysine binding proteins. J Biomol Screening. 2010; 15:6271.

20. Burton PS, Conradi RA, Ho NFH, Hilgers AR, Borchardt RT. How structural features influence the biomembrane permeability of peptides. J Pharm Sci. 1996; 85:1336-1340. [PubMed: 8961149]

21. Ren C, Morohashi K, Plotnikov AN, Jakoncic J, Smith SG, Li J, Zeng L, Rodriguez Y, Stojanoff V, Walsh M, Zhou MM. Small-molecule modulators of methyl-lysine binding for the CBX7 chromodomain. Chem Biol. 2015; 22:161-168. [PubMed: 25660273]

22. Hopkinson RJ, Walport LJ, Munzel M, Rose NR, Smart TJ, Kawamura A, Claridge TD, Schofield CJ. Is JmjC oxygenase catalysis limited to demethylation? Angew Chem Int Ed Engl. 2013; 52:7709-7713. [PubMed: 23788451]

23. Ren C, Smith SG, Yap K, Li S, Li J, Mezei M, Rodriguez Y, Vincek A, Aguilo F, Walsh MJ, Zhou MM. Structure-guided discovery of selective antagonists for the chromodomain of polycomb repressive protein CBX7. ACS Med Chem Lett. 2016; 7:601-605. [PubMed: 27326334]

24. Goldman SD, Funk RS, Rajewski RA, Krise JP. Mechanisms of amine accumulation in, and egress from, lysosomes. Bioanalysis. 20091:1445-1459.

25. Van Dyke RW, Faber ED, Meijer DK. Sequestration of organic cations by acidified hepatic endocytic vesicles and implications for biliary excretion. J Pharmacol Exp Ther. 1992; 261:1-11. [PubMed: 1348536]

26. Copeland RA. The drug-target residence time model: a 10-year retrospective. Nat Rev Drug Discovery. 2016; 15:87-95. [PubMed: 26678621]

27. Barnash KD, Lamb KN, Stuckey JI, Norris JL, Cholensky SH, Kireev DB, Frye SV, James LI. Chromodomain ligand optimization via target-class directed combinatorial repurposing. ACS Chem Biol. 2016; doi: 10.1021/acschembio.6b00415

\section{Abbreviations}

CBX2 chromobox homolog protein 2

CBX4 chromobox homolog protein 4

CBX5 chromobox homolog protein 5

CBX6 chromobox homolog protein 6

CBX7 chromobox homolog protein 7

CBX8 chromobox homolog protein 8

HP1 Heterochromatin protein 1

Kme methyl-lysine

PRC1 Polycomb repressive complex 1

SPR surface plasmon resonance

Pc Polycomb

CDYL2 chromodomain protein, Y-like 2

DMSO dimethyl sulfoxide 


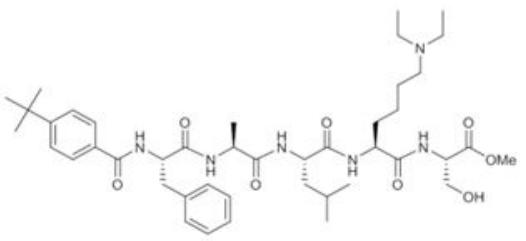

\begin{tabular}{|c|c|c|c|c|}
\hline \multicolumn{2}{|c|}{ Kme Reader Domain } & \multirow[t]{2}{*}{1 (UNC3866) } & \multirow{2}{*}{$\begin{array}{c}\text { Interaction } \\
\text { with PRC1 } \\
\left.P_{c} B_{00}\right]\end{array}$} & \multirow{2}{*}{$\begin{array}{r}\text { UNC3866 } \\
\text { ITC } K_{\mathrm{d}}(\mu \mathrm{M}) \\
1.8 \pm 0.21\end{array}$} \\
\hline $\mathrm{CB} \times 2$ & Chromo | & & & \\
\hline CBX4 & Chromo & & $P_{\mathrm{P}} \mathrm{BO}=\mathrm{s}$ & $0.094 \pm 0.017$ \\
\hline CBX6 & Chromo & PcBor & & $0.610 \pm 0.0078$ \\
\hline CBX7 & Chromo L & $\mathrm{PCBO}_{\mathrm{CO}}$ & & $0.097 \pm 0.0024$ \\
\hline CBX8 & Chromo & Pc Bor & & $1.2 \pm 0.021$ \\
\hline CDY1 & Chromo & & & $6.3 \pm 0.92$ \\
\hline CDYL1b & Chromo & & & $0.91 \pm 0.076$ \\
\hline CDYL2 & Chromo & & 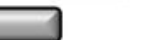 & $0.85 \pm 0.076$ \\
\hline
\end{tabular}

Figure 1. Compound 1 and its chromodomain targets

(Top) Structure of 1, a cell-active peptidic antagonist of CBX and CDY chromodomains. (Bottom) Domain maps of human CBX and CDY chromodomains as annotated in Uniprot. The $K_{\mathrm{d}}$ of compound $\mathbf{1}$ as determined by ITC for each of the chromodomains is listed. 
a

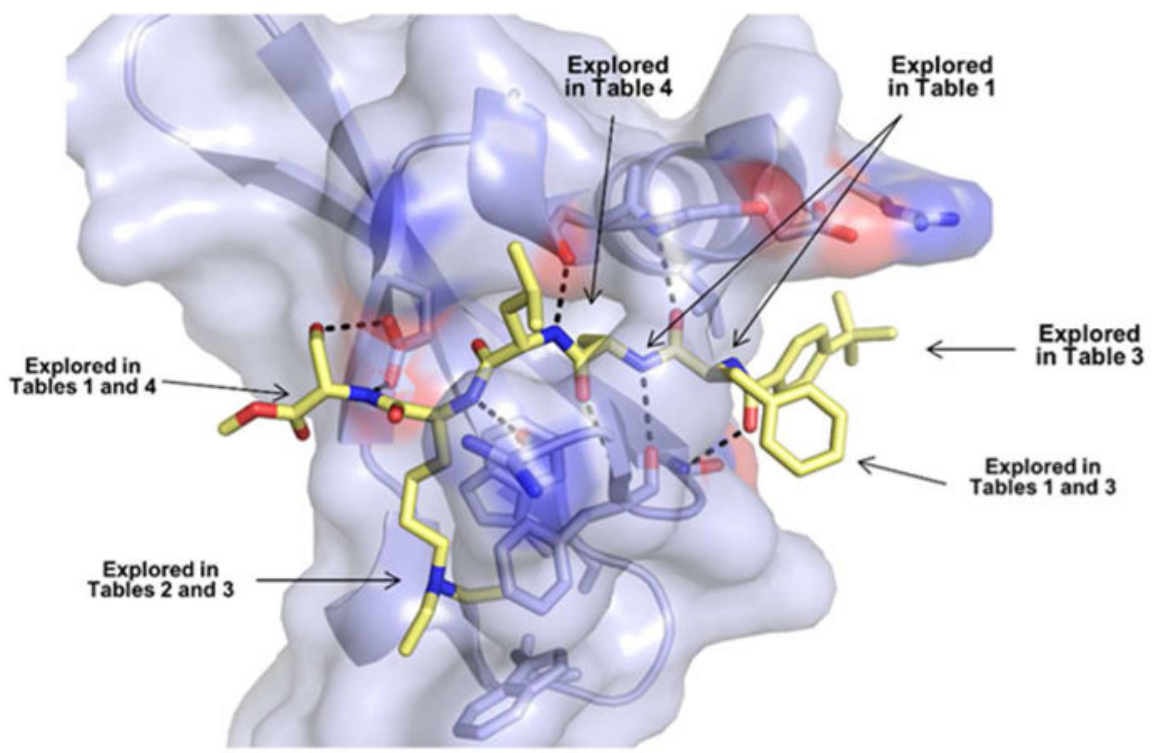

b

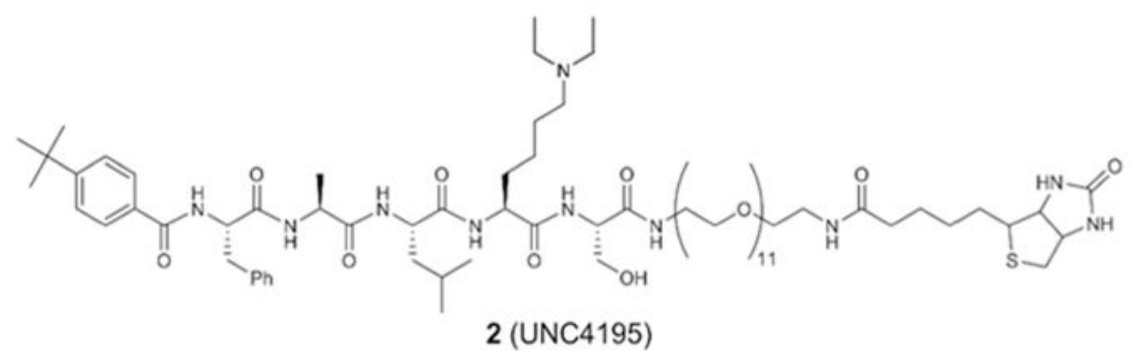

Figure 2. (a) X-ray crystal structure of compound 1 bound to CBX7 (PDB 5EPJ)

Compound $\mathbf{1}$ (yellow) binds in an extended groove along the surface of the chromodomain of CBX7 (light blue). The interaction involves multiple hydrogen bonds (black dashes) between CBX7 and the backbone amides of $\mathbf{1}$. The high affinity of this interaction is driven by hydrophobic contacts between the $t$-butyl group of $\mathbf{1}$ and the side chains of Leu48, Asp49 and Arg51. The different regions of compound $\mathbf{1}$ explored in this study are indicated. (b) Structure of compound $\mathbf{2}$. The biotin affinity tag was added to the C-terminus of $\mathbf{1}$ which is solvent exposed, and $\mathbf{2}$ retains high affinity for CBX7. 

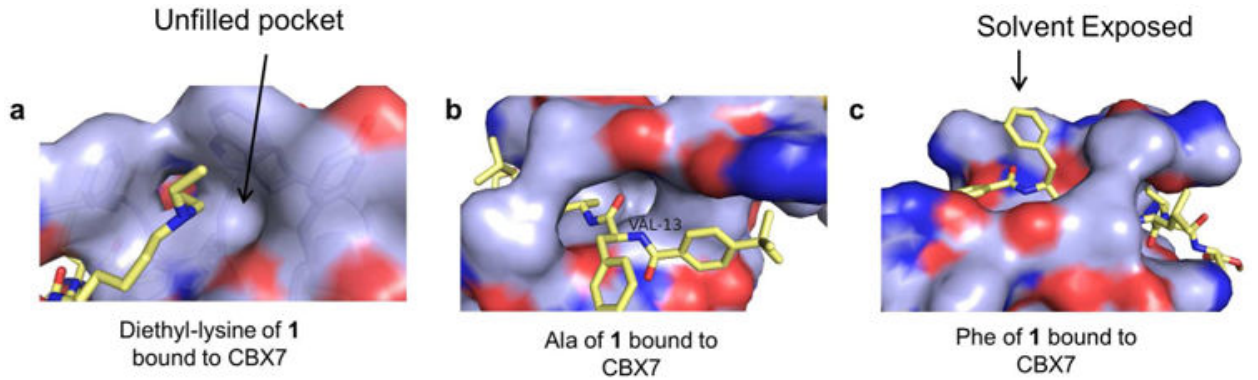

Figure 3. Compound 1 bound to CBX7 (PDB 5EPJ)

(a) Interaction of the $\mathrm{CBX} 7$ aromatic cage with diethyl-lysine. (b) Binding pocket of $\mathrm{CBX} 7$ occupied by the Ala side chain of $\mathbf{1}$. (c) Position of Phe of $\mathbf{1}$ when bound to CBX7. 

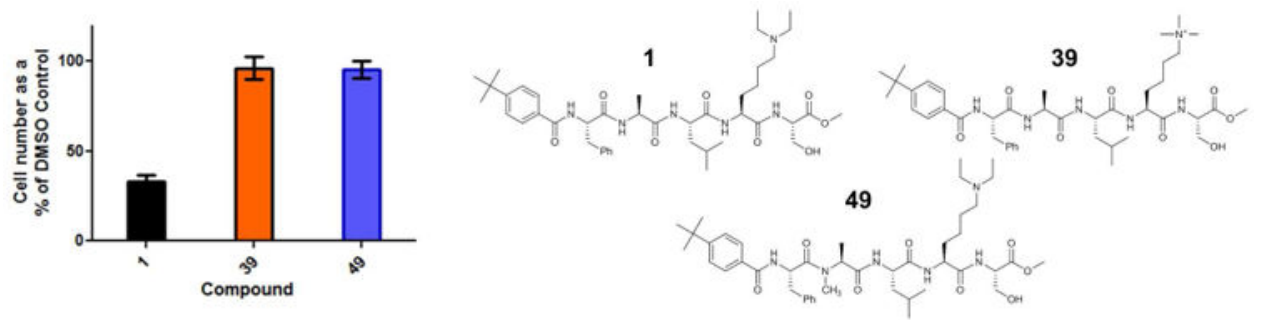

Figure 4. Effect of compounds 1, 39, and 49 on PC3 cell proliferation

Cells were treated for 72 hours with the indicated compounds $(30 \mu \mathrm{M})$ or DMSO, fixed and DAPI stained. Cell counts were numerated using high content microscopy and normalized to DMSO control $(\mathrm{n} \geq 4)$. 


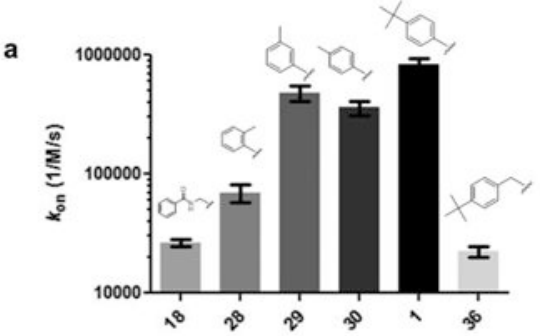

b

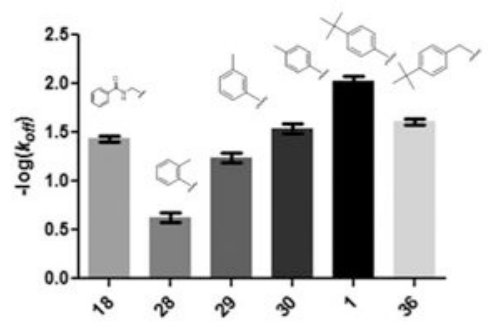

C
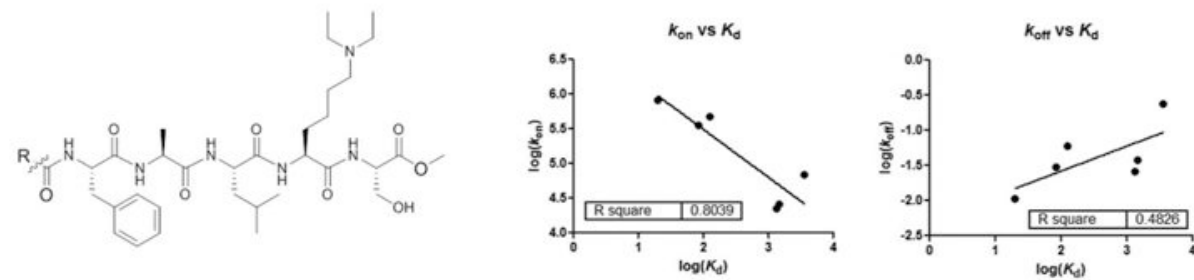

Figure 5.

(a) Comparison of compound-CBX7 associates rates. (b) Comparison of compound-CBX7 dissociation rates. Negative log values of the dissociation rates (1/s) are shown. Data are reported as the mean \pm the standard error of the mean $(n \geq 5)$. (c) CBX7 binding affinities of compounds with $\mathrm{N}$-terminal variations are better correlated with the association rates as compared to the dissociation rates. The log transformations of $k_{\text {on }}(1 / \mathrm{M} / \mathrm{s}), k_{\text {off }}(1 / \mathrm{s})$ and $K_{d}$ $(\mathrm{nM})$ are plotted. 


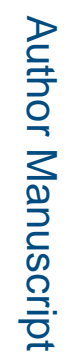

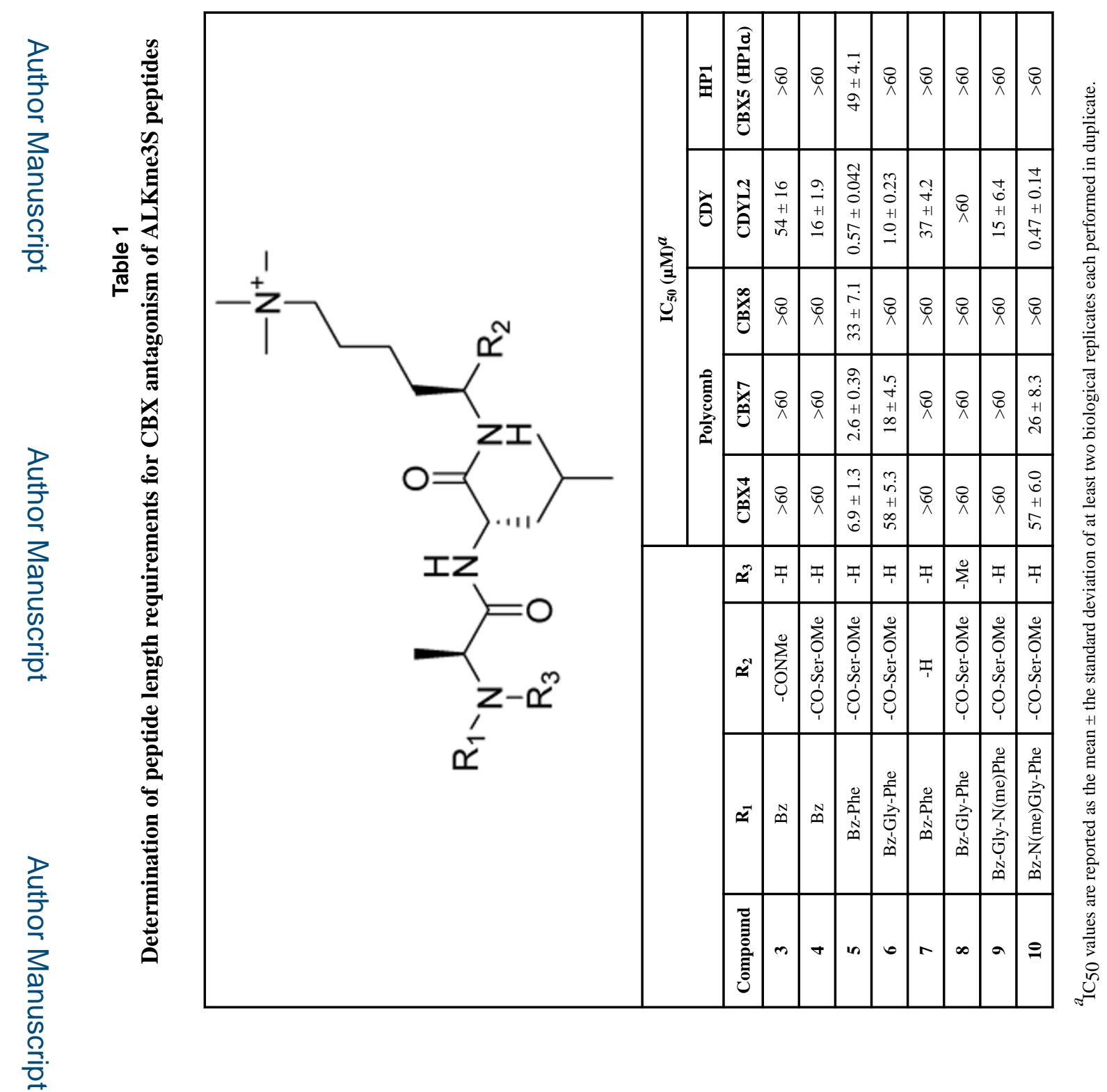

JMed Chem. Author manuscript; available in PMC 2017 October 13. 


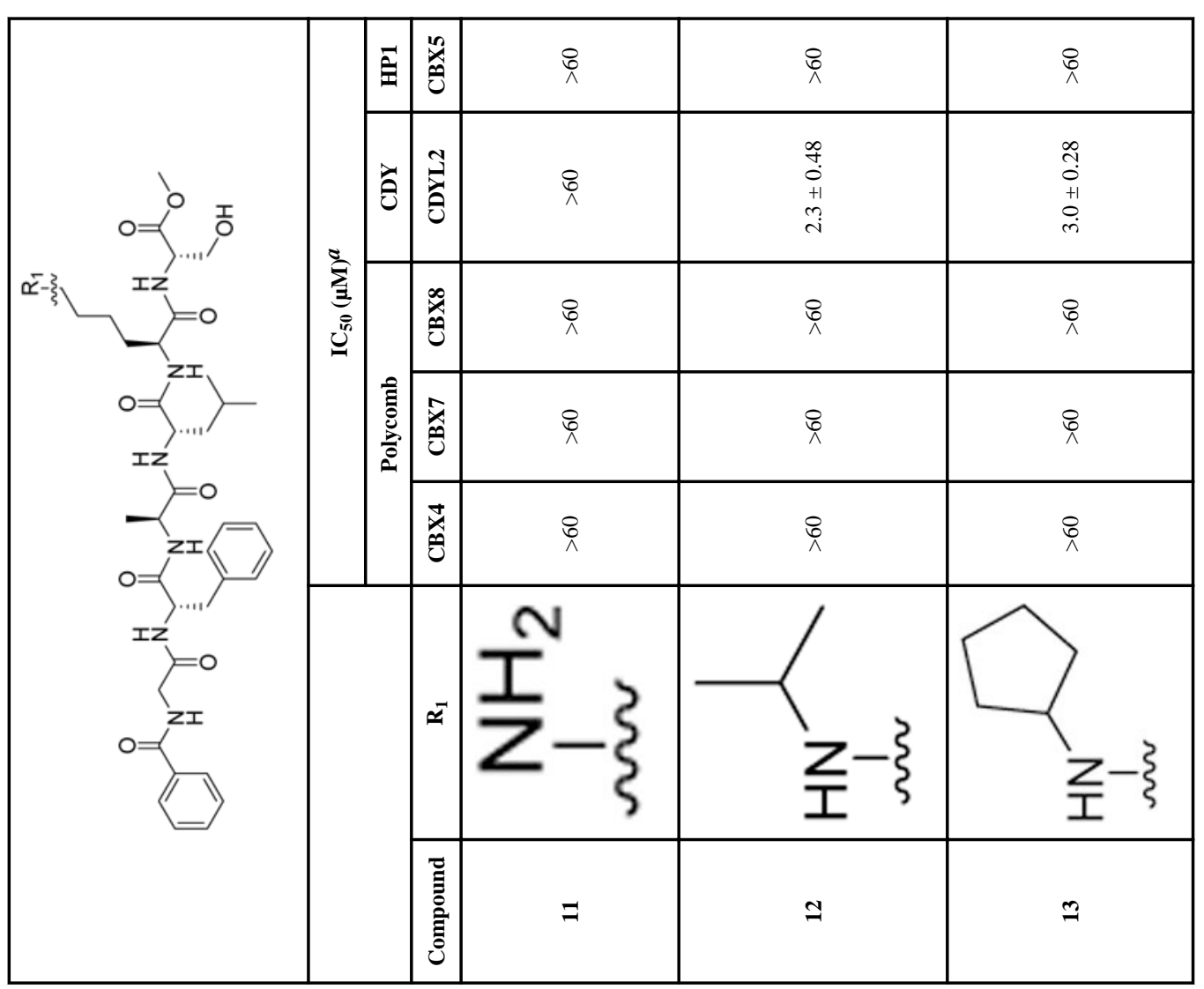

JMed Chem. Author manuscript; available in PMC 2017 October 13. 


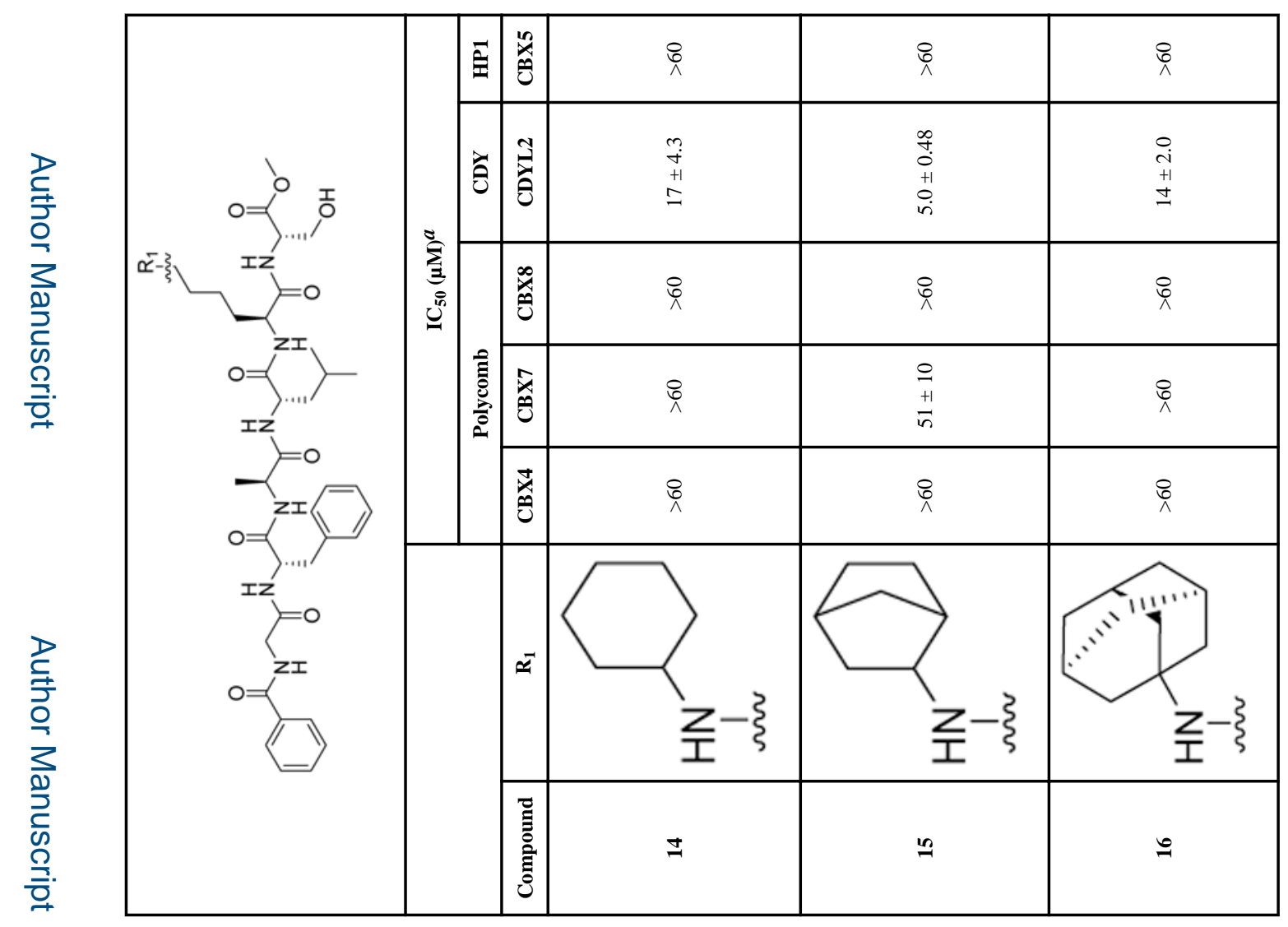

로을

로을 


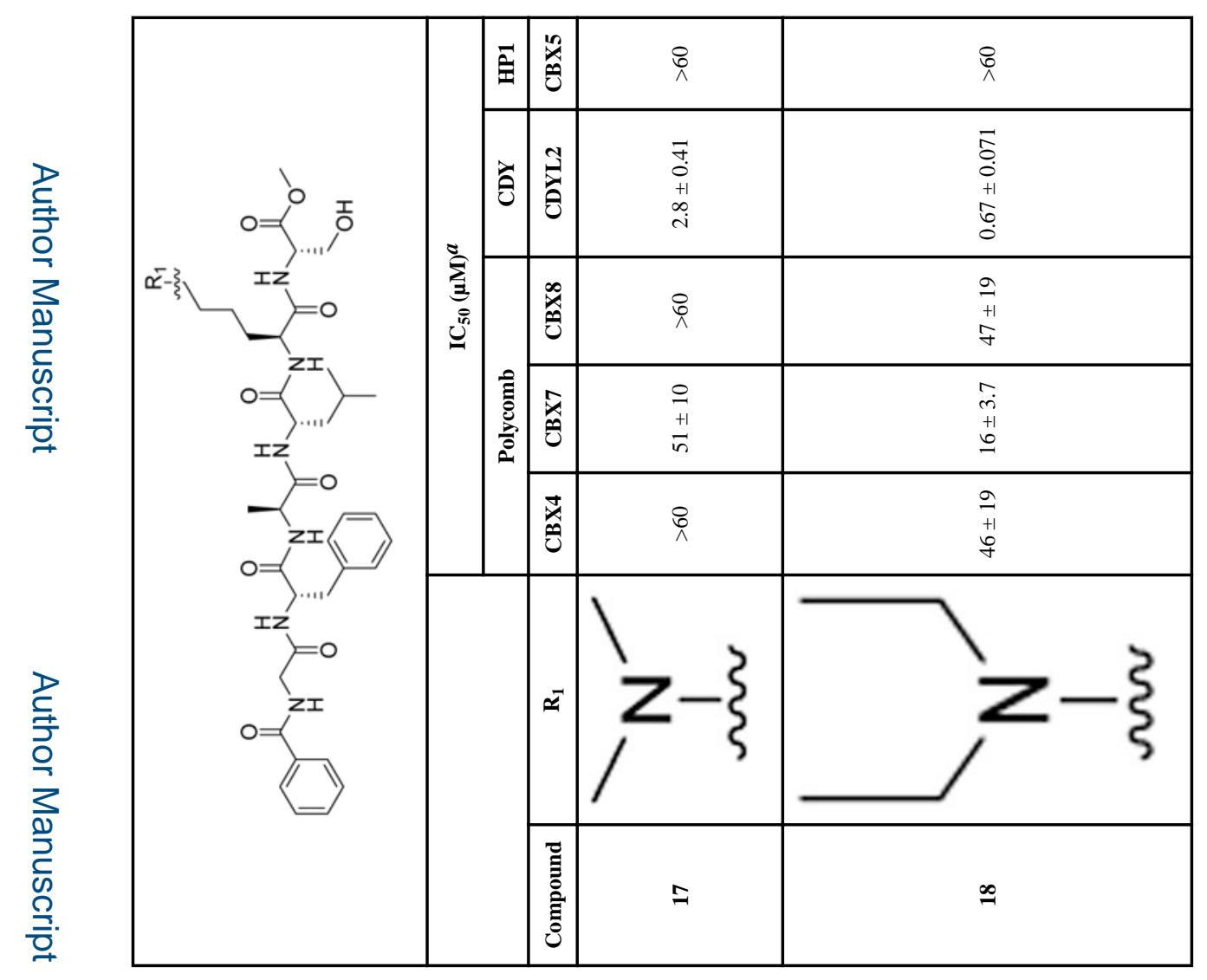

로을

를 


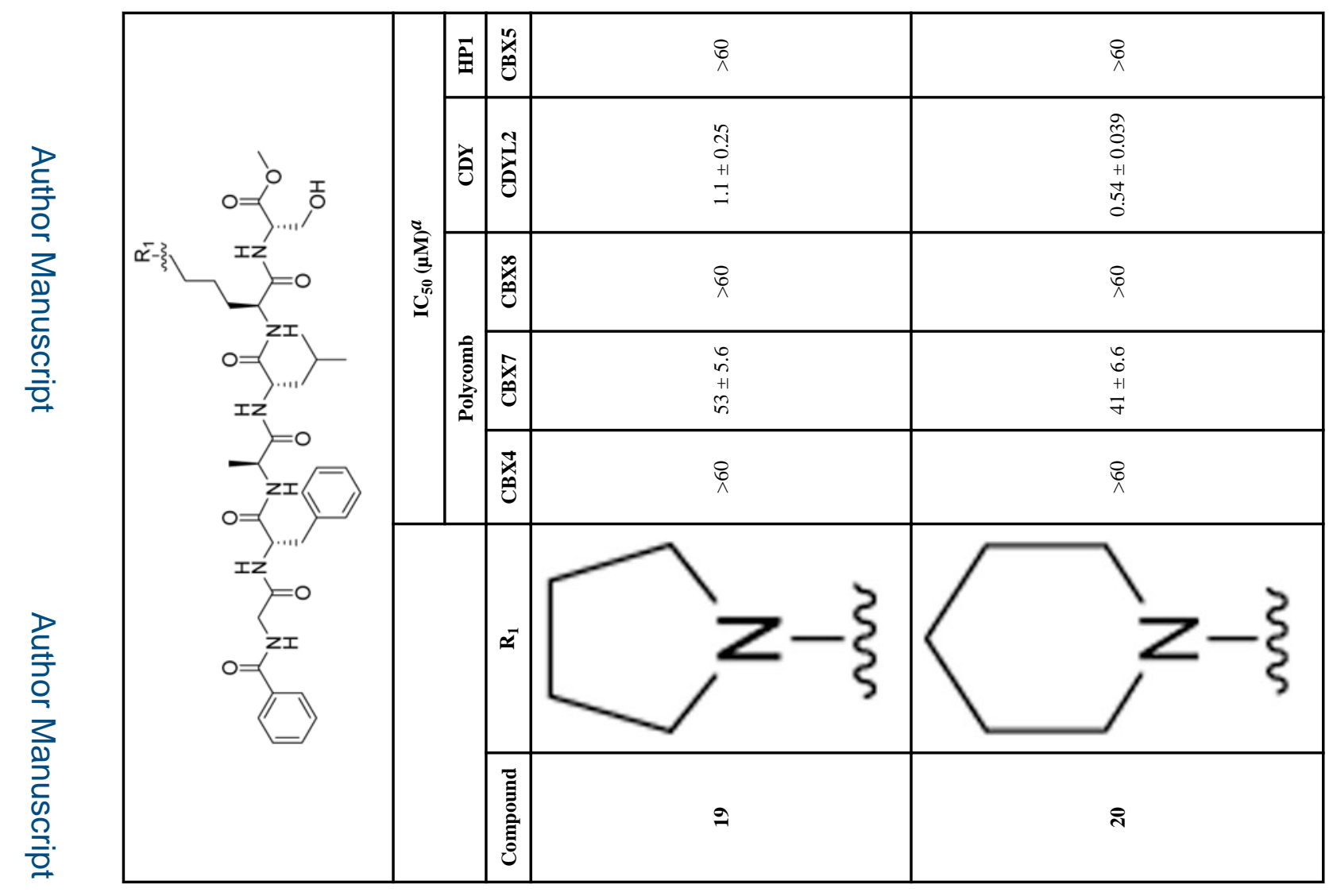

옹

로을 


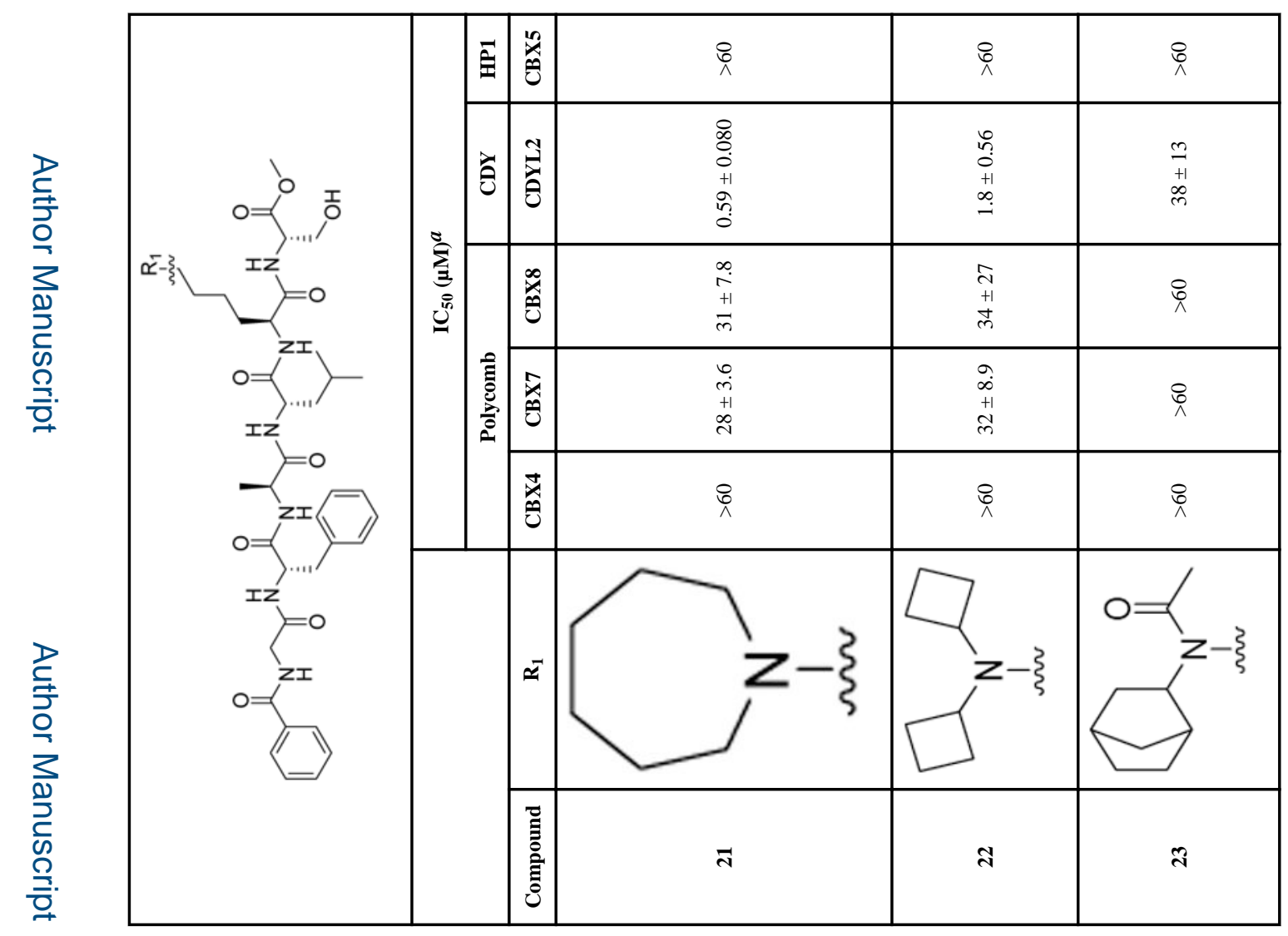

로을

로을 

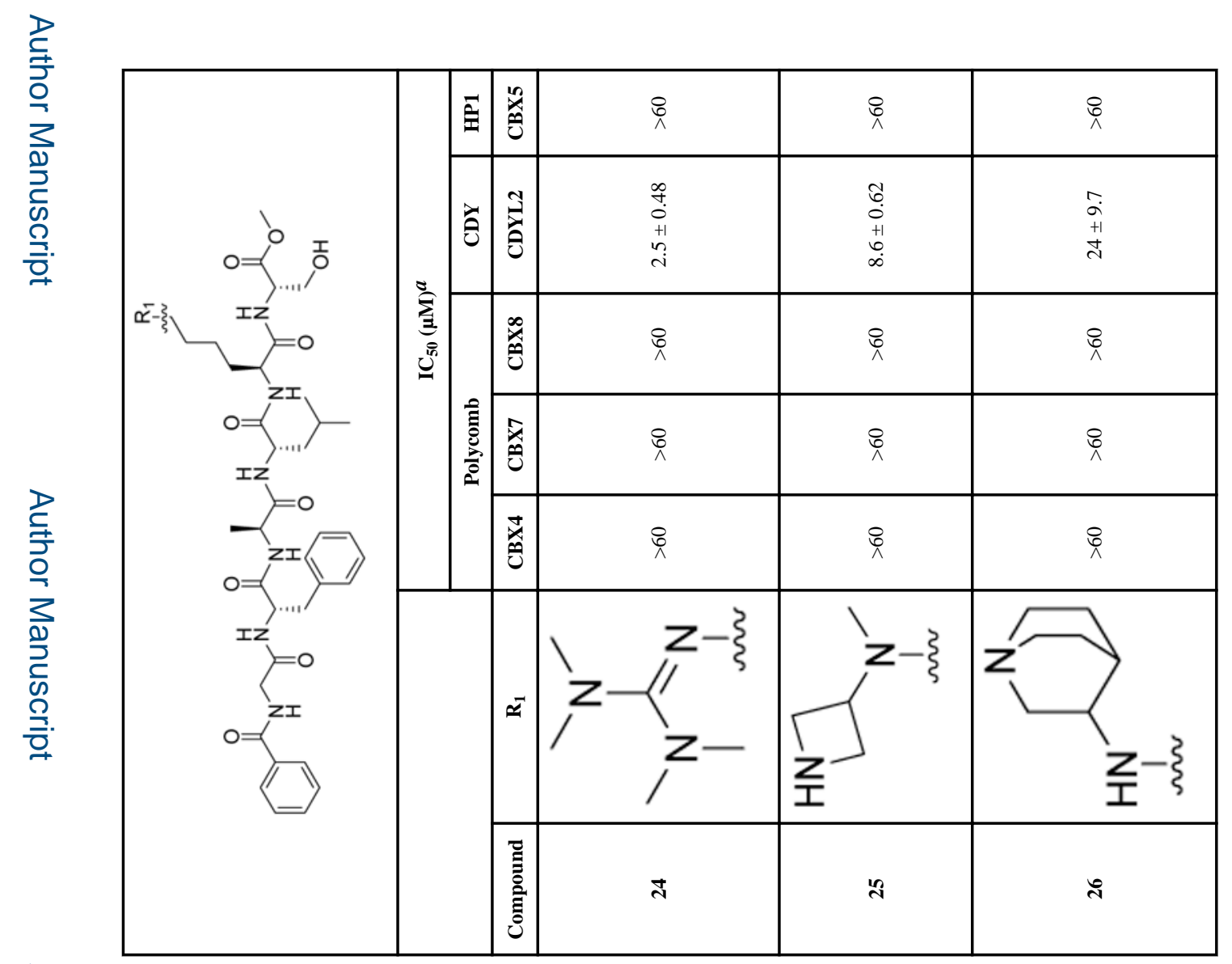

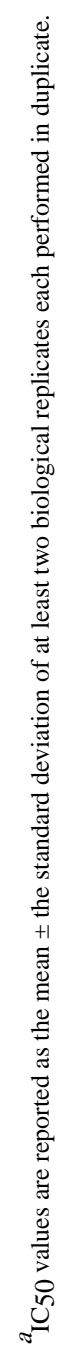

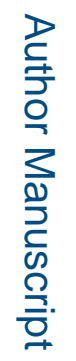

로을 


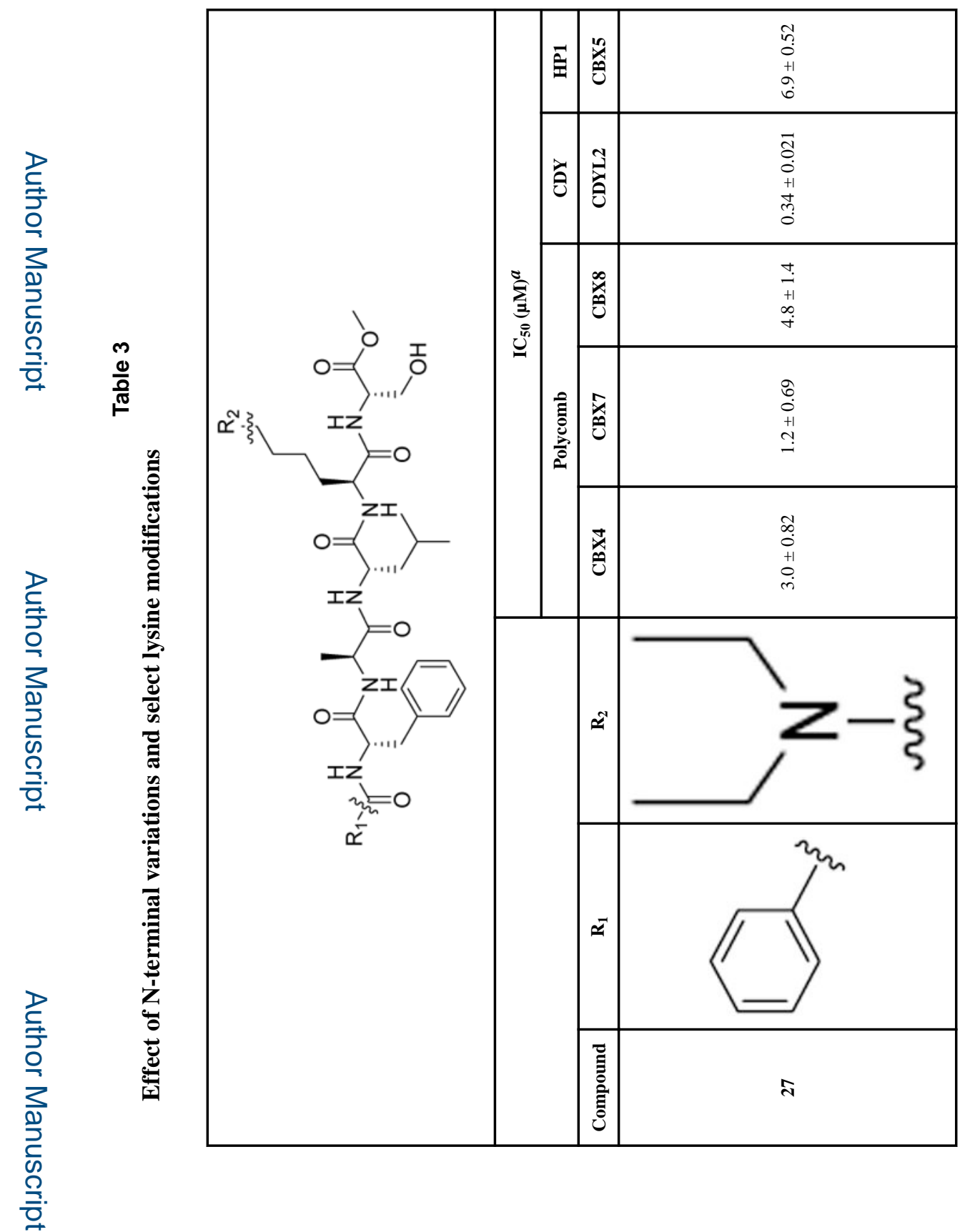

JMed Chem. Author manuscript; available in PMC 2017 October 13. 


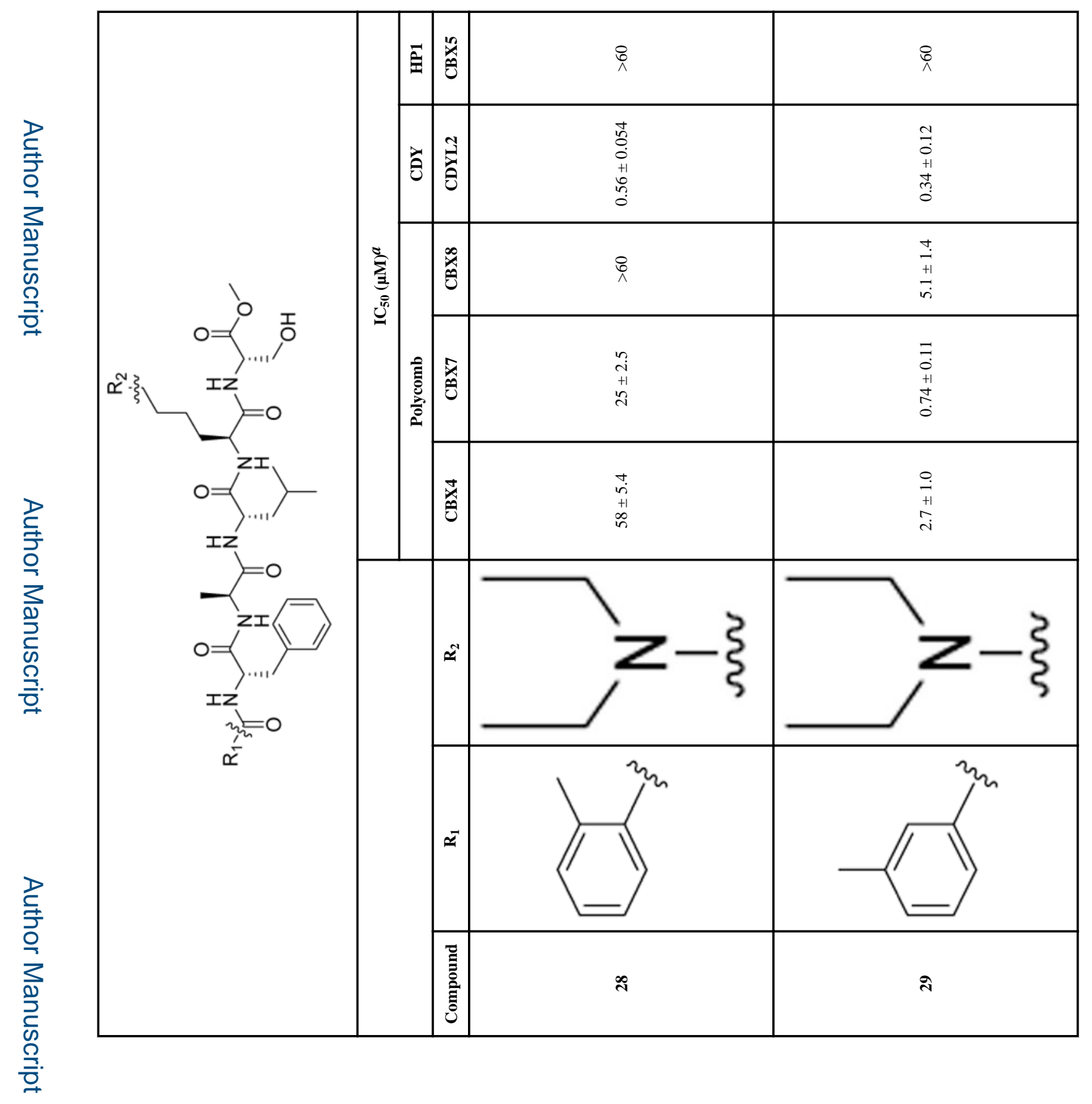

로을 


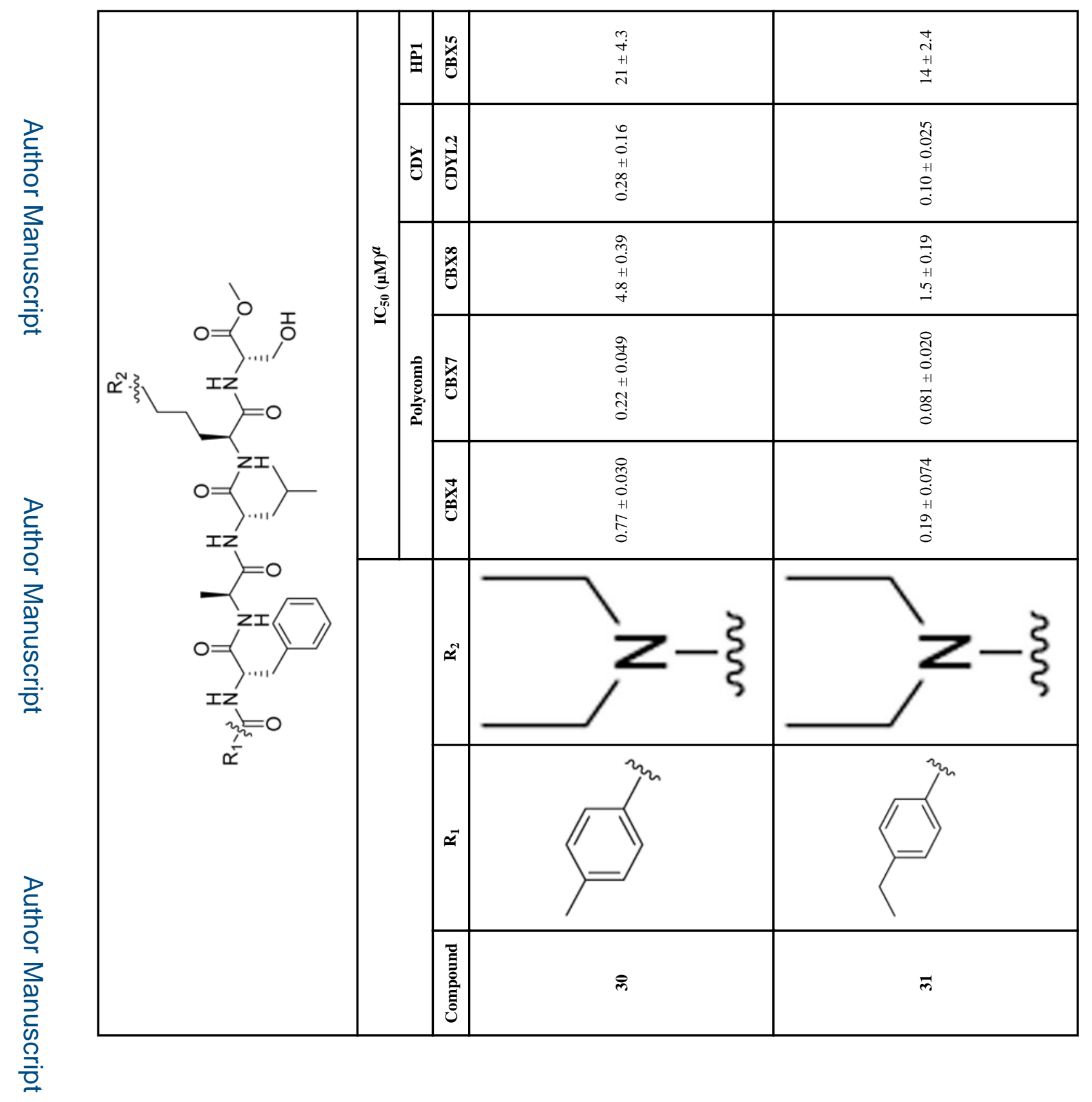

로을 


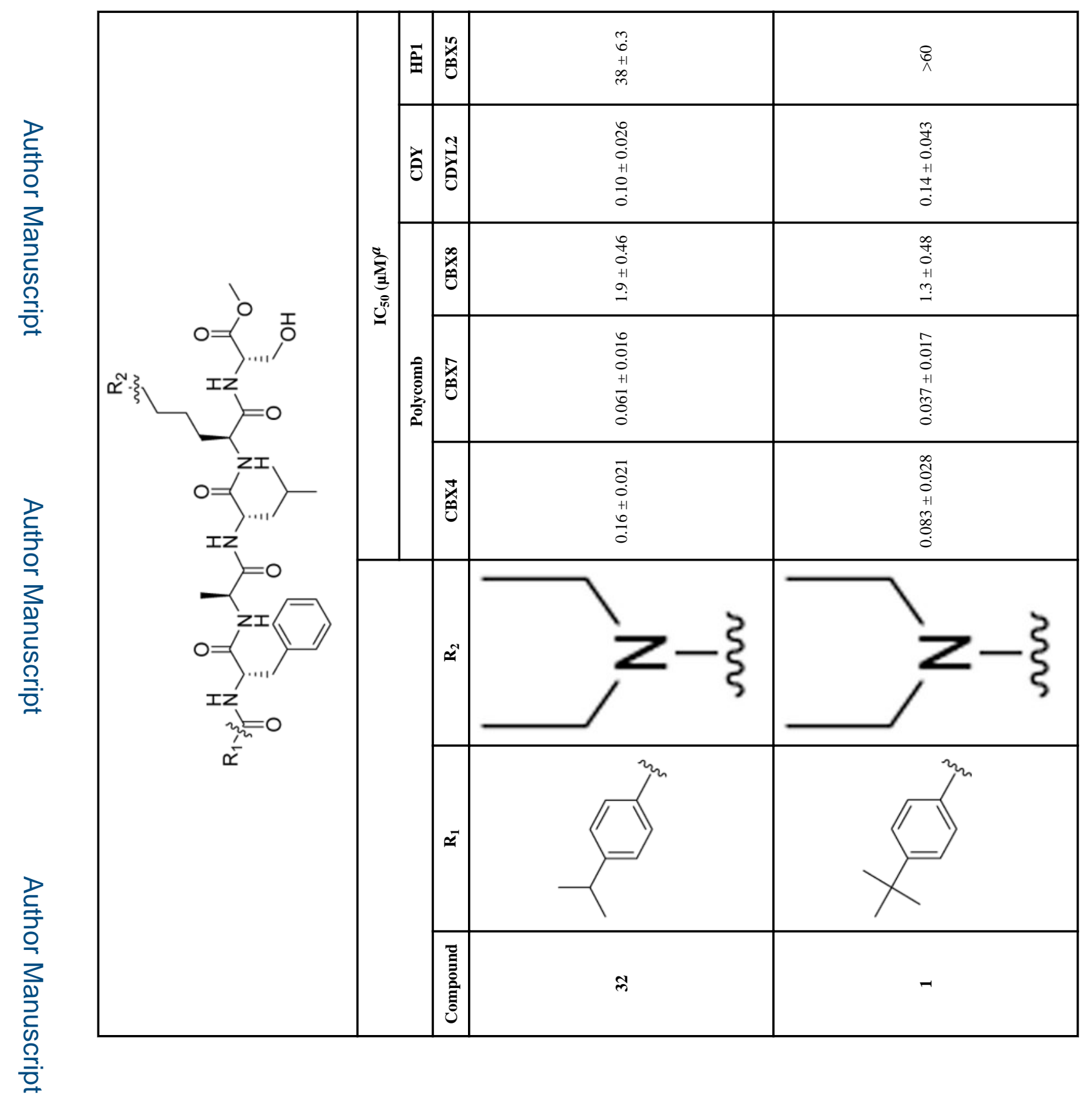

로을 


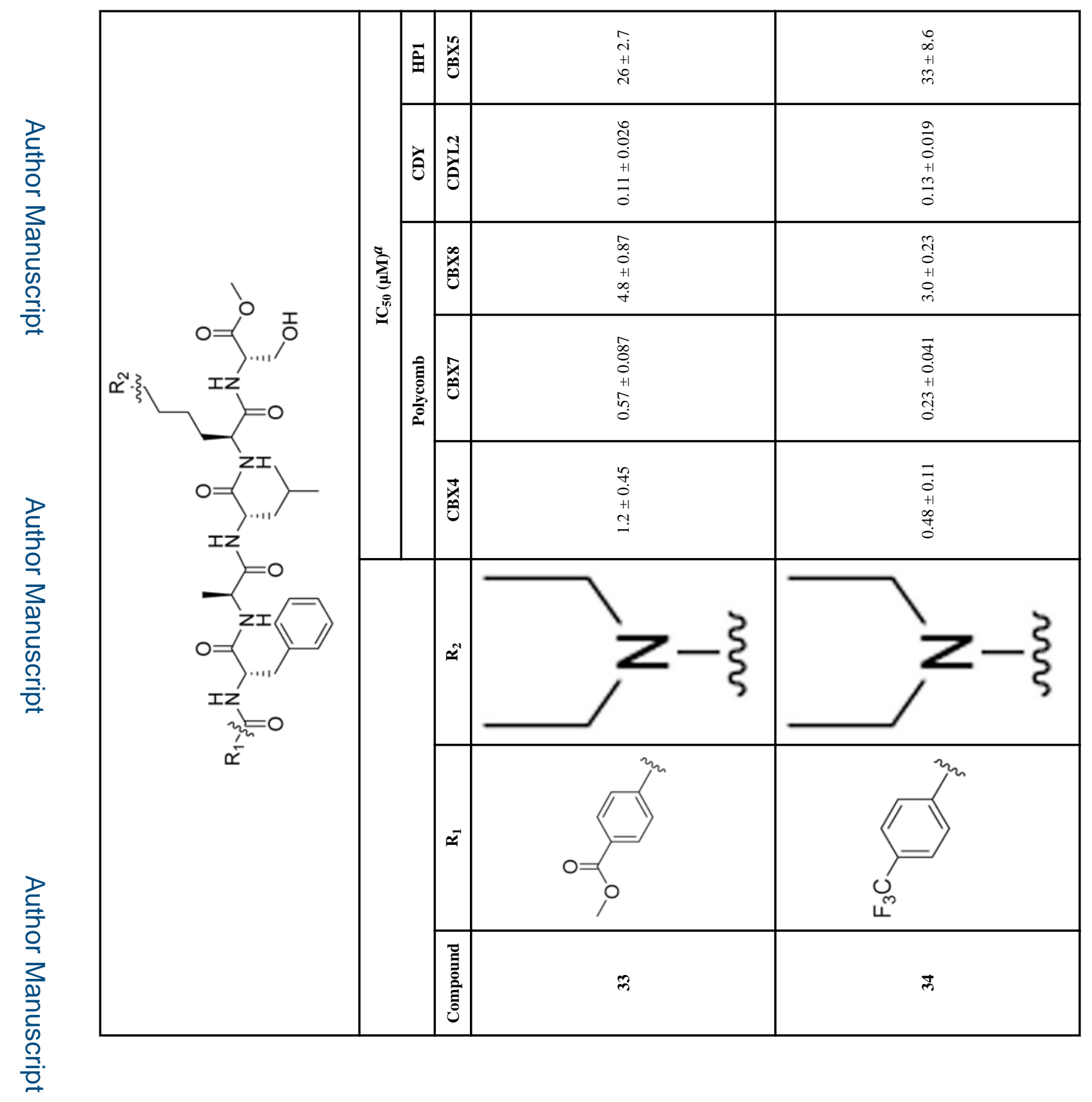

로을 


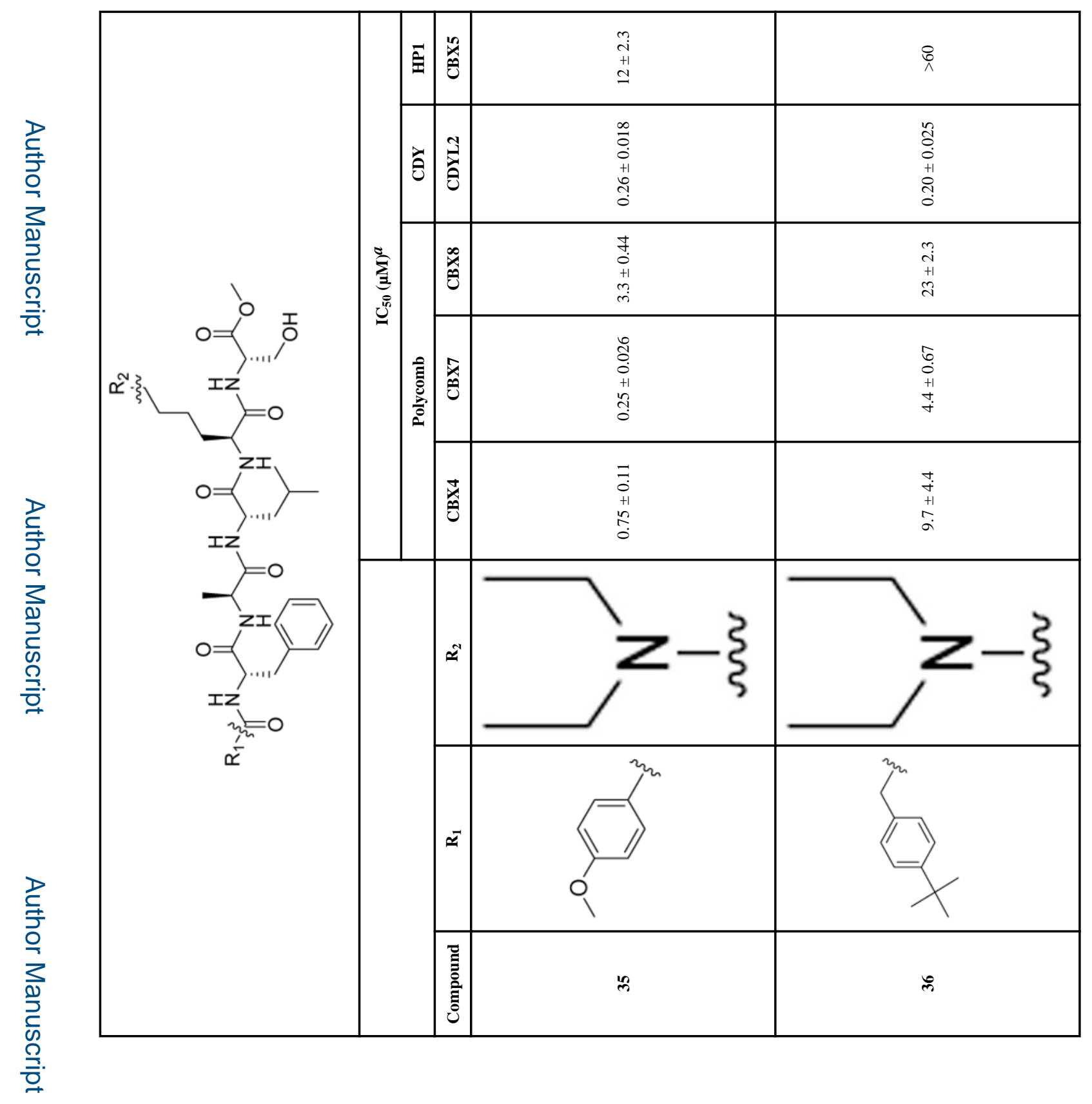

로을 


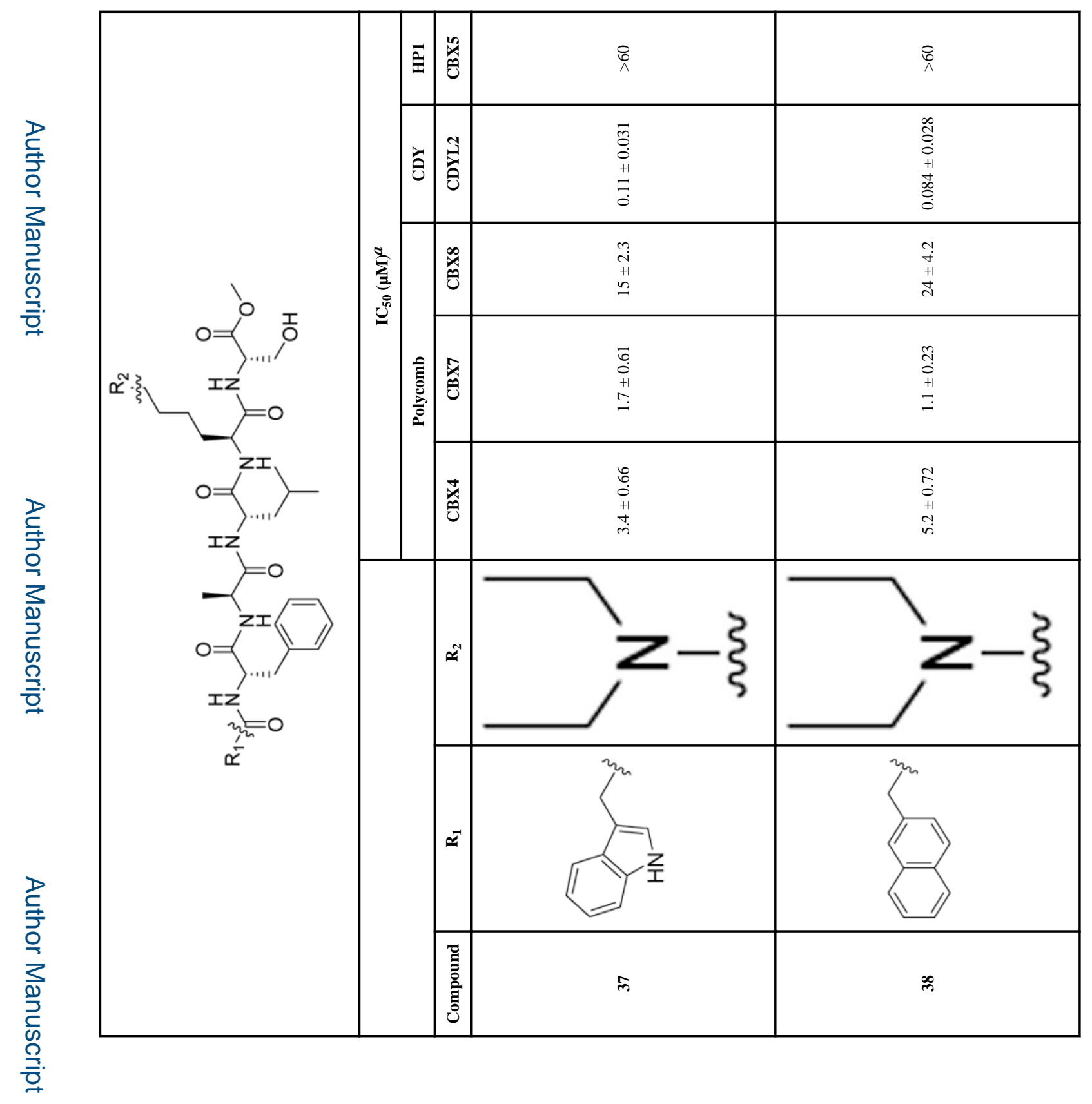

로을 


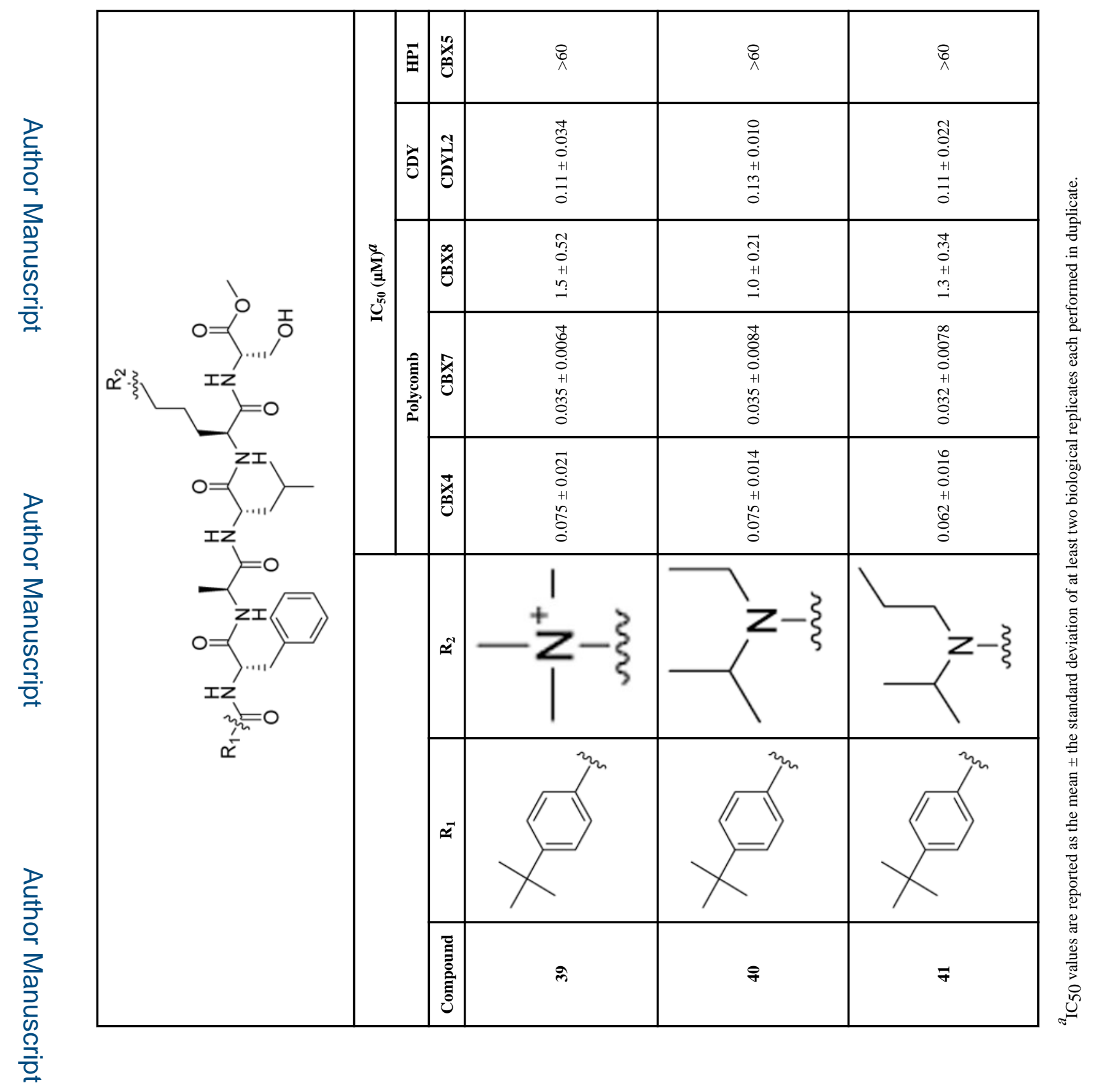

로을 


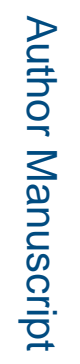

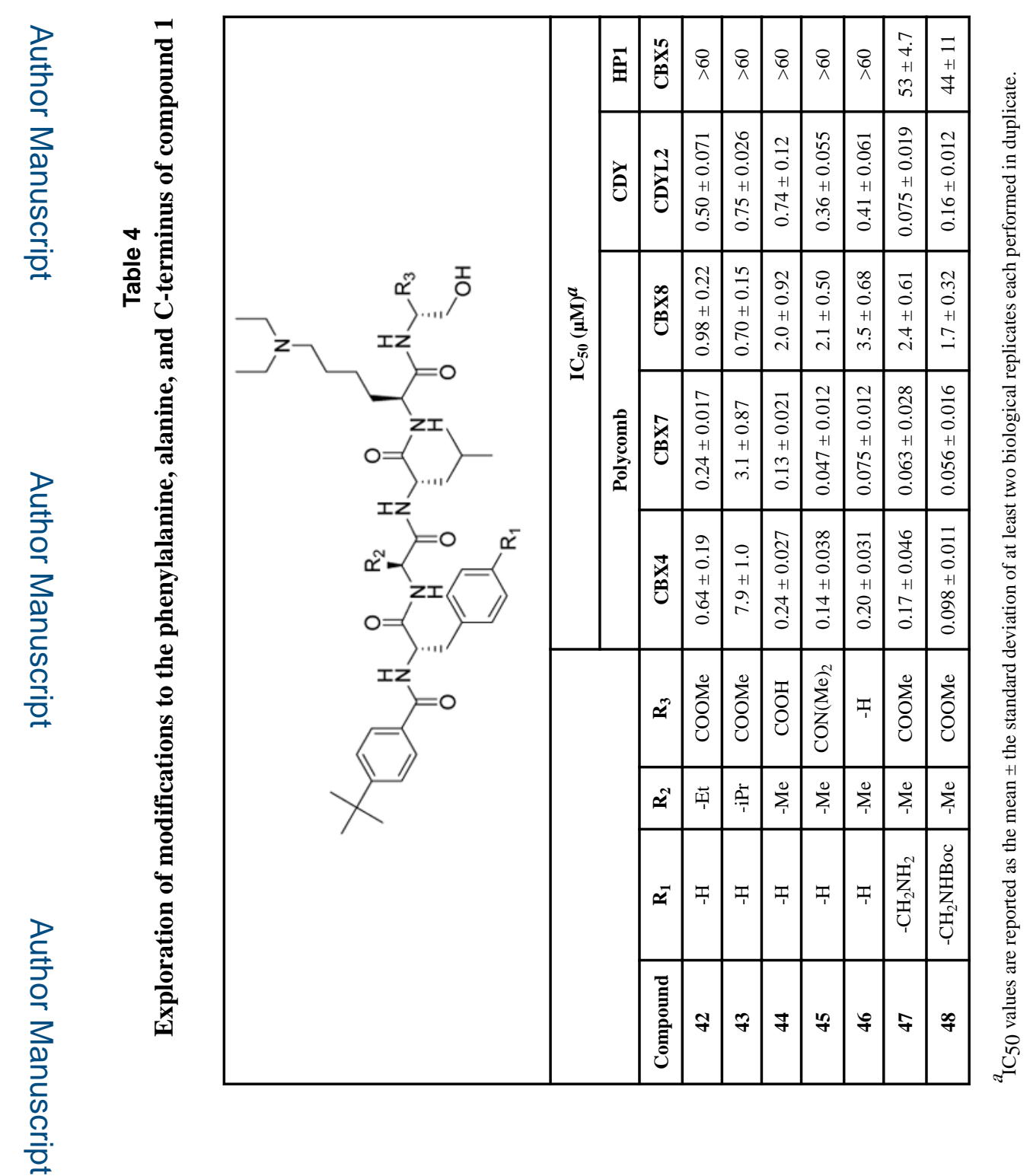

JMed Chem. Author manuscript; available in PMC 2017 October 13. 